\title{
MMSE Design of Time and Color Multiplexing Codes
}

\author{
Tsung-Han Chan, Kui Jia, Eliot Wycoff, and Yi Ma
}

\begin{abstract}
Illumination multiplexing has proven itself to be a valuable tool in image quality improvement for many computer vision and graphics applications, provided that its limitations on photon noise and saturation are properly tackled. Currently, multiplexing codes are constructed according to the maximum signal-to-noise ratio (SNR), and they are primarily employed for time multiplexing, a technique that requires the number of measurements $M$ to be equal to the number of illumination sources $N$. In this work, we propose an illumination multiplexing method based on minimum mean square errors (MMSEs) for the more general setting of both time and color multiplexing performed together, with $K M \geq N$, where $K$ is the number of color channels. Under the umbrella of the proposed MMSE formulation, the conventional maximum SNR approach can be thought of as a special case of the MMSE design. The formulated MMSE problem is a difficult non-convex problem, but it can be approximated by sequential semi-definite (convex) programming and a 1-D exhaustive search. The proposed formulation and algorithm can be readily specialized to max-SNR and/or time multiplexing designs, thereby giving the optimized codes a much broader scope of application. Computer simulations show that the conventional max-SNR design is suboptimal to the proposed MMSE design, though both see significant quality improvements as $M$ increases. Experiments also demonstrate the effectiveness and superiority of the proposed method in illuminating various objects.
\end{abstract}

Index Terms-Optical multiplexing, maximum signal-to-noise ratio (SNR), minimum mean square error (MMSE), sequential convex approximation, time and color.

\section{INTRODUCTION}

$\mathbf{O}$ PTICAL multiplexing can be traced back to the late 1970s to the field of spectrometry [1] in which studies were performed with a single detector simultaneously receiving signals from disparate spectral bands that had been previously encoded. The goal of multiplexing is to improve the quality of demultiplexed, single spectral band signals. An analogous

Manuscript received April 22, 2013; revised November 19, 2013; accepted December 30, 2013. Date of publication January 14, 2014; date of current version February 07, 2014. The associate editor coordinating the review of this manuscript and approving it for publication was Dr. Andrzej Cichocki. This study was partially supported by a research grant for the Human Sixth Sense Programme at the Advanced Digital Sciences Center from Singapore's Agency for Science, Technology and Research (A*STAR), and by the funding of NSF CCF 09-64215 and NSF IIS 11-16012.

T.-H. Chan, K. Jia, and E. Wycoff are with Advanced Digital Sciences Center, Singapore 138632, Singapore (e-mail: thchan@ieee.org; Chris.jia@adsc.com.sg; wycoffe@gmail.com).

$\mathrm{Y} . \mathrm{Ma}$ is with theDepartment of Electrical and Computer Engineering, University of Illinois at Urbana-Champaign, Urbana, IL 61821 USA, and also with Microsoft Research Asia, Beijing, China, 100080 (e-mail: yima@illinois.edu).

Color versions of one or more of the figures in this paper are available online at http://ieeexplore.ieee.org.

Digital Object Identifier 10.1109/TSP.2014.2300032 multiplexing concept was brought into the domain of computer vision and graphics by Schechner et al. in 2003 [2]; in this work, objects were illuminated by multiple sources from different directions, and the resulting images were computationally demultiplexed with the intent of acquiring single-light source images of maximum signal-to-noise ratio (SNR) ${ }^{1}$. This max-SNR multiplexing scheme was later employed in numerous applications, such as scene recovery [3], object relighting [4], and photometric stereo [5]-[7], and it has proven to be valuable in SNR improvement.

The SNR boost for single-light source images is expected to have a positive impact on computer vision applications such as face recognition [8] in which gathering training face images under arbitrary illuminations via multiplexing is expected to improve recognition rates. In photometric stereo [6], [7], estimating the surface normals of objects from demultiplexed, single-light source images can substantially aid in object recognition and 3-D modeling. Moreover, a large number of singlelight source images of a scene can be used to create a new image with arbitrary lighting conditions. Multiplexed illuminations with the relighting technique further help in film postproduction [5]; e.g., the lighting and reflectance of the actor can be designed and modified after the film was shot. However, illumination multiplexing is not always favorable in improving image quality. When considering photon noise along with sensor noise, multiplexing multiple light sources may be counterproductive [9]. Present efforts for designing max-SNR multiplexing codes in the presence of both sensor noise and photon noise include [9]-[11], but they are limited to time multiplexing systems where the number of measurements $(M)$ equals the number of illumination sources $(N)$. Some questions then arise: what can we gain from multiplexing if the number of measurements $M$ is larger than the number of illumination sources $N$ ? How can the multiplexing codes be designed to include color multiplexing in addition to time multiplexing? And further, are there any relevant code design criteria apart from a maximum SNR?

Present efforts have partially addressed the aforementioned limitations of optical multiplexing. Alterman et al. [12] relaxed the limitation that $M=N$ for max-SNR multiplexing for multiplexed fluorescence unmixing, but their problem design additionally has to take into account the natural mixing in fluorescent images, which does not fall into the scope of this paper. Moreover, many existing works have utilized time and color multi-

\footnotetext{
${ }^{1}$ The conventional maximum-SNR approach can be shown to be a type of constrained Minimum Mean Square Errors (MMSE) problem; see Section II. To differentiate it from the generic MMSE design, we keep its name as it is throughout the paper.
} 
plexing for various purposes, for instance: [4] employing cyan, magenta and yellow patterns for the illumination of the faces of mannequins; [13] using a cluster of light sources with different spectra and an RGB camera for multispectral imaging; [14] extending time multiplexing with color multiplexing for the capture of dynamic scenes; and [6] utilizing time and color multiplexing for dynamic photometric stereo. Nonetheless, hardly any information exists on how such necessary time and color multiplexing codes could have been optimally constructed.

In this paper, we propose a design of time and color multiplexing codes using the Minimum Mean Square Error (MMSE) criterion for the more general setting of $K M \geq N$, where $K$ denotes the number of color channels and, for example, $K=$ 3 for the case of an RGB color camera. The optimal multiplexing code design problem takes into account the presence of sensor noise and photon noise, as well as the saturation challenge [10], and it can be formulated into a constrained optimization problem. Since the formulated problem unfortunately turns out to be non-convex, we then propose a systematic method, which we call the Illumination Multiplexing Codes (IMC) algorithm, that approximates the problem by sequential convex programming and a 1-dimensional exhaustive search. The proposed MMSE formulation and algorithm can be readily specialized to the problem of conventional max-SNR design and/or time multiplexing, thereby giving codes optimized in such a fashion as to yield a much broader application. Simulations show the merits of our approach: first, the MMSE design yields more multiplexing gain than the conventional max-SNR design, and both significantly improve image quality as $M$ increases; and second, a fewer number of measurements, say $M / K$, used in time and color multiplexing is sufficient for achieving equal performance when using $M$ time multiplexing. The second advantage implies that one could reduce the number of acquisitions by using more color channels, and this could benefit applications where the number of acquired images should be as few as possible, such as dynamic photometric stereo [6]. Our experiments show the effectiveness and superiority of the proposed approach in illuminating various objects, such as Lambertian and non-Lambertian objects.

This paper is based on our conference paper [15] that tackles the design of multiplexing code based on the max-SNR criterion. The main differences are summarized as follows.

- We provide a new MMSE criterion formulation, under which the conventional max-SNR approach [1], [2], [4], [10]-[12] is viewed as a constrained MMSE problem.

- The MMSE-based formulated problem is more general and more difficult than the max-SNR problem [15]. Some additional reformulations and approximations are presented.

- A convergence analysis of the proposed IMC algorithm is discussed, and some pros and cons of the MMSE and max-SNR designs are also elaborated.

- Simulations and experiments with both MMSE and max-SNR designs are conducted. The results show the superiority of the MMSE design over the max-SNR design.

- Objects with Lambertian and non-Lambertian surfaces are used in object illumination experiments.

Organization of this paper: In Section II, we review the background of time multiplexing as well as related work. Section III

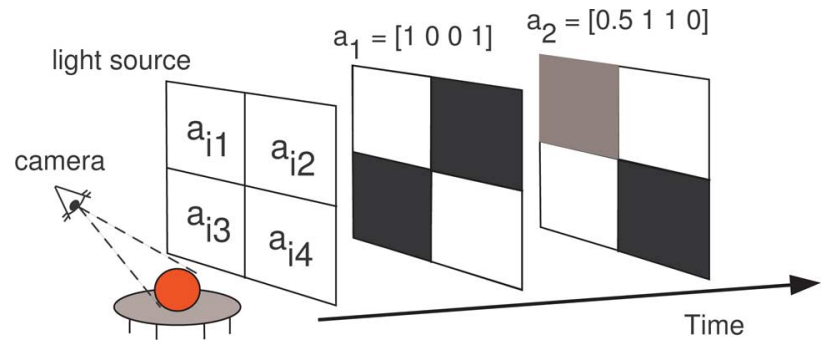

Fig. 1. Illustration of time multiplexing with $N=4$ single-light sources: an object is viewed under varying illumination directions encoded by the elements of $\mathbf{A}$. The row vectors of $\mathbf{A}$ correspond to different illumination multiplexing conducted at different time instances. An element of each row of $\mathbf{A}$, ranging from 0 to 1 , controls the brightness of the corresponding light source.

presents how we use the MMSE criterion to design time and color multiplexing codes and to develop the IMC algorithm. Section IV presents the specialization of the IMC algorithm to max-SNR multiplexing. In Section V, we present some simulation results that demonstrate the viability of the proposed MMSE design and its algorithms and their advantages relative to some other existing algorithms. Section VI gives experimental results with the proposed approach for various examples of object relighting. Finally, conclusions are drawn in Section VII.

\section{BACKGROUND OF TIME MultiPLEXING}

In this section we briefly review the background of time multiplexing from a general-to-specific perspective, and we introduce some related work. To begin with, let us consider the scenario in which a static object with Lambertian surfaces is illuminated by multiple (say $N$ ) diverse single light sources using a time-multiplexed scheme, and let each light source have its own direction from which it illuminates a surface patch of the object. Assuming there are $N$ distinct multiplexed illuminations in total, the captured intensity value at pixel $n$ can be represented by the linear superposition model,

$$
\mathbf{x}[n]=\mathbf{A s}[n]+\mathbf{v}[n], \quad n=1, \ldots, L,
$$

where $\mathbf{x}[n] \in \mathbb{R}^{N}$ is a vector containing the captured light intensity for $N$ different multiplexed illuminations at pixel $n, \mathbf{A} \in \mathbb{R}^{N \times N}$ is a time multiplexing matrix, $\mathbf{s}[n]=\left[s_{1}[n], \ldots, s_{N}[n]\right]^{T}$ denotes a vector comprised of the intensities of the reflected light at the $n$th pixel under $N$ different single light source conditions, $\mathbf{v}[n] \in \mathbb{R}^{N}$ is the measurement noise, and $L$ is the total number of image pixels. The 1-dimensional pixel index $n$ results from a vector transformation of the 2-dimensional image coordinates. Fig. 1 illustrates how time multiplexing is applied to a real scenario.

Time multiplexing in (1) can use either a switching ${ }^{2}$ or a nonswitching strategy. We use the non-switching strategy, where the elements of the matrix $\mathbf{A}$ range over the entire interval from 0 to 1 :

$$
\mathbf{0} \preceq \operatorname{vec}(\mathbf{A}) \preceq \mathbf{1}_{N N} .
$$

\footnotetext{
${ }^{2}$ The elements of $\mathbf{A}$ are binary, belonging to either 0 or 1 .
} 
Here $\preceq$ denotes the componentwise inequality for vectors and the linear matrix inequality for matrices, $\operatorname{vec}(\cdot)$ denotes the vectorization operator, $\mathbf{1}_{N}$ is the $N$-dimensional vector whose entries are each equal to 1 , and $\mathbf{0}$ is the zero vector of proper dimension. To clarify, the notation $\mathbf{1}_{N N}$ is the all-one vector of dimension $N^{2}$. The extreme values 0 and 1 stand for the corresponding light source being completely turned off and turned on, respectively. The values of $\{\mathbf{s}[n]\}_{n=1}^{L}$ depend on the relative positions, orientation and visibility of the object and its light sources. In addition, the noise $\mathbf{v}[n]$ is assumed to be independent and identically distributed (i.i.d.) with zero mean and a covariance matrix satisfying the affine noise model [4], [16]:

$$
\boldsymbol{\Sigma}_{P}=\left(\sigma^{2}+P \rho^{2}\right) \mathbf{I}_{N},
$$

where $\sigma^{2}$ denotes the variance of the signal-independent sensor noise, $\rho^{2}$ denotes the variance of the signal-dependent photon noise, $\mathbf{I}_{N}$ is $N \times N$ the identity matrix, and $P$ is the total energy of the activated light sources at each measurement. The value of $P$ has a direct connection to the time multiplexing matrix $\mathbf{A}$, with the multiplexing power used in each measurement being equal to $P$ :

$$
\mathbf{A} \mathbf{1}_{N}=P \mathbf{1}_{N}
$$

The goal is to jointly devise both the multiplexing matrix $\mathbf{A}$ and the demultiplexing matrix $\mathbf{B} \in \mathbb{R}^{N \times N}$ such that the demultiplexed, single light source illuminations

$$
\begin{array}{r}
\hat{\mathbf{s}}[n]=\mathbf{B}^{T} \mathbf{x}[n]=\mathbf{B}^{T} \mathbf{A} \mathbf{s}[n]+\mathbf{B}^{T} \mathbf{v}[n], \\
n=1, \ldots, L,
\end{array}
$$

have minimum mean square errors (MMSE) with respect to $\mathbf{s}[n]$, denoted by

$$
\begin{aligned}
\mathcal{E}_{\mathrm{t}}(\mathbf{A}, \mathbf{B}, P) & \triangleq \operatorname{Tr}\left(\mathbb{E}\left((\hat{\mathbf{s}}[n]-\mathbf{s}[n])(\hat{\mathbf{s}}[n]-\mathbf{s}[n])^{T}\right)\right) \\
& \left.=\operatorname{Tr}\left(\mathbf{B}^{T} \mathbf{A}-\mathbf{I}_{N}\right) \mathbf{R}\left(\mathbf{B}^{T} \mathbf{A}-\mathbf{I}_{N}\right)^{T}+\mathbf{B} \boldsymbol{\Sigma}_{P} \mathbf{B}^{T}\right),
\end{aligned}
$$

where $\operatorname{Tr}(\cdot)$ is the trace operator, $\mathbb{E}$ is the expectation operator, and $\mathbf{R}=\mathbb{E}\left(\mathbf{s}[n] \mathbf{s}[n]^{T}\right)$ stands for the (positive semi-definite) correlation matrix of the demultiplexed images. Here, the demultiplexed images and the noise are assumed to be statistically mutually independent; i.e., $\mathbb{E}\left(\mathbf{s}[n] \mathbf{v}[n]^{T}\right)=\mathbf{0}$. By defining the feasible set of $\mathbf{A}$ from (2) and (4)

$$
\mathcal{A}_{P}=\left\{\mathbf{A} \in \mathbb{R}^{N \times N} \mid \mathbf{0} \preceq \operatorname{vec}(\mathbf{A}) \preceq \mathbf{1}_{N N}, \mathbf{A} \mathbf{1}_{N}=P \mathbf{1}_{N}\right\},
$$

the design of the multiplexing codes is equivalent to solving the following constrained optimization problem:

$$
\left\{\mathbf{A}^{\star}, \mathbf{B}^{\star}, P^{\star}\right\}=\arg \min _{\mathbf{A} \in \mathcal{A}_{P}, \mathbf{B} \in \mathbb{R}^{N \times N}, P \in \mathbb{R}} \mathcal{E}_{\mathrm{t}}(\mathbf{A}, \mathbf{B}, P) .
$$

Compared to the scenario with single-source acquisition, i.e., $\mathbf{A}=\mathbf{I}_{N}$, the time multiplexing gain is easily computed to be

$$
G_{\mathrm{t}} \triangleq \sqrt{\frac{\operatorname{Tr}\left(\boldsymbol{\Sigma}_{1}\right)}{\mathcal{E}_{\mathrm{t}}\left(\mathbf{A}^{\star}, \mathbf{B}^{\star}, P^{\star}\right)}},
$$

where $\boldsymbol{\Sigma}_{1}$ is given by (3) with $P=1$.

Problem (9) is a difficult non-convex problem. Early efforts [1], [2], [4], [10]-[12] primarily focused on handling the MMSE problem (9) with the added constraint of $\mathbf{B}^{T} \mathbf{A}=\mathbf{I}_{N}$ :

$$
\begin{array}{r}
\left\{\mathbf{A}^{\sharp}, \mathbf{B}^{\sharp}, P^{\sharp}\right\}=\arg \min _{\mathbf{A} \in \mathcal{A}_{P}, \mathbf{B} \in \mathbb{R}^{N \times N}, P \in \mathbb{R}} \mathcal{E}_{\mathrm{t}}(\mathbf{A}, \mathbf{B}, P) \\
\text { s.t. } \mathbf{B}^{T} \mathbf{A}=\mathbf{I}_{N},
\end{array}
$$

Here we use $\sharp$ to differentiate the solution of (11) from the optimal solution of the generic MMSE problem, denoted as *. In such a case, the unique solution of the demultiplexing matrix can be easily determined to be $\mathbf{B}^{\sharp}=\left(\mathbf{A}^{-1}\right)^{T}$, as $\mathbf{A}$ and $\mathbf{B}$ are both square. Hence, problem (11) can be further simplified to

$$
\left\{\mathbf{A}^{\sharp}, P^{\sharp}\right\}=\arg \min _{\mathbf{A} \in \mathcal{A}_{P}, P \in \mathbb{R}} \operatorname{Tr}\left(\left(\mathbf{A}^{T} \boldsymbol{\Sigma}_{P}^{-1} \mathbf{A}\right)^{-1}\right) .
$$

Clearly, the above problem minimizes the sum of the noise power in $\hat{\mathbf{s}}[n]=\mathbf{s}[n]+\mathbf{A}^{-1} \mathbf{v}[n]$ (from (5)) or, equivalently, maximizes its signal-to-noise ratio (SNR). Problem (12) originates from a constrained MMSE formulation, but to differentiate the general MMSE design (9), we name this instance as the maximum-SNR design. From (10), the multiplexing gain for the max-SNR design is then computed as

$$
G_{\mathrm{t}}=\sqrt{\frac{\operatorname{Tr}\left(\boldsymbol{\Sigma}_{1}\right)}{\operatorname{Tr}\left(\left(\left(\mathbf{A}^{\sharp}\right)^{T} \boldsymbol{\Sigma}_{P^{\sharp}}^{-1} \mathbf{A}^{\sharp}\right)^{-1}\right)}} .
$$

\section{A. Related Work}

Present efforts for handling the max-SNR problem (11) were briefly reviewed, and they have been categorized by the conditions under which the following methods were developed.

1) Absence of Photon Noise: Optical multiplexing techniques were first investigated in the field of spectrometry [1]. The optimal multiplexing codes for problem (12) have been shown to be the S-matrix [1], [2], [4] where photon noise is absent, i.e., $\rho^{2}=0$. The S-matrix can be readily constructed based on the Hadamard codes of length $N+1$ for some $N$ such that $(N+1) / 4$ is an integer. Hadamard multiplexing is a switch multiplexed method that completely turns on $P^{\sharp}=(N+1) / 2$ light sources during each measurement. Using Hadamard multiplexing, the multiplexing gain is $G_{\mathrm{t}}=(N+1) /(2 \sqrt{N})$ [1]. Thus it is clear that by using multiplexing the greater the number of illumination sources $N$, the better the obtained image quality will be.

2) Presence of Photon Noise: When photon noise comes into play, Hadamard multiplexing is no longer optimal [5]. Mutting [9] looked into the effect of photon noise on Hadamard multiplexing and derived new multiplexing codes based on twolevel autocorrelation sequences. These multiplexing codes have shown their advantages when there is photon noise, but they are 
only available for a very limited set of values of $N$ and for a limited range of noise variances $\left\{\sigma^{2}, \rho^{2}\right\}$. Thus, Ratner et al. [10] constructed new time multiplexing codes for any $N$ and noise variance $\left\{\sigma^{2}, \rho^{2}\right\}$. They handled problem (12) for a given $P$ by using the projected gradient method which, unfortunately, is easily susceptible to getting stuck in the local optima during the solution search; hence, they also devised a higher level optimization procedure for the method to escape from local optima [10]. After collecting all the optimal objective values of problem (12) for all of the $P$ 's, the optimal $P^{\sharp}$ was then selected to be the one with the minimum objective value.

3) Saturation: Saturation is another problem encountered during illumination multiplexing, and it tends to occur when the object of interest is illuminated by numerous light sources. When the captured image intensity is saturated - that is, the linear superposition model (1) is violated —one should either reduce the exposure time or decrease the total energy $P$ for each measurement. [2] and [4] have proven that the latter is better than the former. Thus, to counter the saturation issue, Ratner et $a l$. have added the constraint,

$$
P \leq P_{\text {sat }},
$$

where $P_{\text {sat }}$ is the threshold beyond which the captured image becomes saturated.

Despite the success of the above methods for problem (11), there still remain some notable limitations:

- The current optimal multiplexing codes are limited to time multiplexing only.

- The current multiplexing system must be a determined system, i.e., $\mathbf{A} \in \mathbb{R}^{M \times N}$ and $M=N$.

- There are no available methods for handling the MMSE design problem (9), a problem which, in principle, will yield a multiplexing gain larger than or equal to that of the max-SNR design. ${ }^{3}$

Thus, some natural questions arise. First, can the code-design problem for time multiplexing be extended to that of both time and color multiplexing? Second, what happens if the multiplexing system captures a greater number of measurements? And third, can one develop a method for handling the MMSE design problem (9)? In the next section, we formulate an MMSE design problem for a time and color multiplexing system without requiring $M=N$. Some advantages of employing the MMSE design for over-determined time and color multiplexing systems will also be shown and discussed.

\section{The MMSE Design of TIME AND COLOR MULTIPLEXING CODES}

Time and color multiplexing has been utilized for relighting [4], multispectral imaging [13], and image capturing under varying illumination conditions [6], [14]; but none of these applications specifically elaborate how to optimally devise the time and color multiplexing codes whether they be based on the MMSE or max-SNR criteria. In this section, we introduce a model for time and color multiplexing, and we present how the MMSE codes for time and color multiplexing can be designed.

\footnotetext{
${ }^{3}$ Since the constraint set of (11) is a subset of (9), the MMSE design would yield a $\hat{\mathbf{s}}[n]$ that leads the $\operatorname{MSE}\|\mathbb{E}(\hat{\mathbf{s}}[n]-\mathbf{s}[n])\|_{2}^{2}$ to be smaller than that of the max-SNR design.
}

The formulation and the algorithm can then be easily tailored to time multiplexing.

Time and color multiplexing is a natural extension of time multiplexing alone. Noticeable difference lies in that three elements corresponding to red, green, and blue channels are used to control the color and brightness of each light source. As reported in [14], the captured time-multiplexing images of a static Lambertian object via RGB channels should fulfill the following linear superposition model:

$\mathbf{x}_{c}[n]=\alpha_{c}[n] \mathbf{A}_{c} \mathbf{s}[n]+\mathbf{v}_{c}[n] n=1, \ldots, L, c \in\{r, g, b\}$

where $\mathbf{x}_{r}[n], \mathbf{x}_{g}[n], \mathbf{x}_{b}[n] \in \mathbb{R}^{M}$ denote the $M$ time multiplexed image intensity at pixel $n$, recorded via red, green, and blue channels, respectively; $\alpha_{r}[n], \alpha_{g}[n], \alpha_{b}[n]$ $\in \mathbb{R}$ represent illumination-independent, normalized RGB intensities of the material at image pixel $n$ satisfying $\alpha_{r}[n]+\alpha_{g}[n]+\alpha_{b}[n]=1, n=1, \ldots, L$; $\mathbf{A}_{r}, \mathbf{A}_{g}, \mathbf{A}_{b} \in \mathbb{R}^{M \times N}$ are the time-multiplexing matrices for the red, green, and blue channels, respectively; $\mathbf{s}[n] \in \mathbb{R}^{N}$ corresponds to the various single-light source illuminations at pixel $n$; and $\mathbf{v}_{r}[n], \mathbf{v}_{g}[n], \mathbf{v}_{b}[n] \in \mathbb{R}^{M}$ denote the noise measured in the RGB channels, respectively. The noise covariance matrices for RGB channels are usually diverse, depending on the specification of the color camera used, i.e., $\boldsymbol{\Sigma}_{P}^{c}=\left(\sigma_{c}^{2}+P \rho_{c}^{2}\right) \mathbf{I}_{M}, c \in\{r, g, b\}$. Noise correlations across the RGB channels ${ }^{4}$, caused by the Bayer filter and color interpolation, are assumed to be modelled as a function of $P$; i.e., $\Theta_{P}^{i, j} \in \mathbb{R}^{M \times M}$ for $i, j \in\{r, g, b\}$ and $i \neq j$. Also, the material colors and the noise covariance matrices can be practically acquired in the camera calibration phase, and they are assumed to be known hereafter. The details of how to estimate those parameters can be found in Section VI-A.

We first reformulate the model in (15). Moving the effects of the material colors $\alpha_{r}[n], \alpha_{g}[n], \alpha_{b}[n], n=1, \ldots, L$ into the noise term, and stacking the RGB counterparts as a column vector, we obtain

$\mathbf{y}[n] \triangleq\left[\begin{array}{c}\mathbf{x}_{r}[n] / \alpha_{r}[n] \\ \mathbf{x}_{g}[n] / \alpha_{g}[n] \\ \mathbf{x}_{b}[n] / \alpha_{b}[n]\end{array}\right]=\mathbf{F} \mathbf{s}[n]+\mathbf{w}[n], \quad n=1, \ldots, L$,

where $\mathbf{F}=\left[\mathbf{A}_{r}^{T}, \mathbf{A}_{g}^{T}, \mathbf{A}_{b}^{T}\right]^{T} \in \mathbb{R}^{3 M \times N}$ is the time and color multiplexing matrix, and $\mathbf{w}[n]=\left[\mathbf{v}[n]_{r}^{T} / \alpha_{r}[n], \mathbf{v}[n]_{g}^{T} / \alpha_{g}[n], \mathbf{v}[n]_{b}^{T} / \alpha_{b}[n]\right]^{T} \in \mathbb{R}^{3 M}$ is the noise with the following covariance matrix,

$$
\boldsymbol{\Lambda}_{P}=\left[\begin{array}{ccc}
\frac{\sum_{n=1}^{L} \boldsymbol{\Sigma}_{P}^{r}}{\alpha_{n}^{2}[n] L} & \frac{\sum_{n=1}^{L} \Theta_{P}^{r, g}}{\alpha_{r}[n] \alpha_{g}[n] L} & \sum_{n=1}^{L} \Theta_{P}^{r, b} \\
\sum_{n=1}^{L} \boldsymbol{\Theta}_{P}^{g, r} & \sum_{n=1}^{L} \boldsymbol{\Sigma}_{P}^{g} & \left.\sum_{n=1}^{L} \boldsymbol{\Theta}_{P}^{g}\right], b \\
\frac{\alpha_{g}[n] L}{\alpha_{g}[n] \alpha_{r}[n] L} & \frac{\boldsymbol{\alpha}_{g}[n] \alpha_{b}[n] L}{\alpha_{g}[n] L} \\
\frac{\sum_{n=1}^{L} \boldsymbol{\Theta}_{P}^{b, r}}{\alpha_{b}[n] \alpha_{r}[n] L} & \frac{\sum_{n=1}^{L} \boldsymbol{\Theta}_{P}^{b, g}}{\alpha_{b}[n] \alpha_{g}[n] L} & \frac{\sum_{n=1}^{L} \boldsymbol{\Sigma}_{P}^{b}}{\alpha_{b}^{2}[n] L}
\end{array}\right] .
$$

Assuming that $3 M \geq N$, the demultiplexed single-source illumination $\mathbf{s}[n]$, can be written as

$$
\begin{array}{r}
\hat{\mathbf{s}}[n]=\mathbf{G}^{T} \mathbf{y}[n]=\mathbf{G}^{T} \mathbf{F} \mathbf{s}[n]+\mathbf{G}^{T} \mathbf{w}[n], \\
n=1, \ldots, L,
\end{array}
$$

\footnotetext{
${ }^{4}$ Any sophisticated model of the noise correlation across RGB channels is applicable to the following proposed algorithm, as long as it can be explicitly expressed as a function of $P$.
} 
where $\mathbf{G} \in \mathbb{R}^{3 M \times N}$ is a time and color demultiplexing matrix. In the same spirit as the MMSE design for time multiplexing (9), the MMSE design of time and color multiplexing codes can be carried out by minimizing the MMSE

$$
\begin{aligned}
& \mathcal{E}_{\mathrm{tc}}(\mathbf{F}, \mathbf{G}, P) \\
& \quad=\operatorname{Tr}\left(\left(\mathbf{G}^{T} \mathbf{F}-\mathbf{I}_{N}\right) \mathbf{R}\left(\mathbf{G}^{T} \mathbf{F}-\mathbf{I}_{N}\right)^{T}+\mathbf{G} \boldsymbol{\Lambda}_{P} \mathbf{G}^{T}\right) \\
& \quad=\operatorname{Tr}\left(\mathbf{G}^{T}\left(\mathbf{F} \mathbf{R} \mathbf{F}^{T}+\boldsymbol{\Lambda}_{P}\right) \mathbf{G}-\mathbf{G}^{T} \mathbf{F R}-\mathbf{R} \mathbf{F}^{T} \mathbf{G}+\mathbf{R}\right),
\end{aligned}
$$

under the constraint that

$$
\begin{aligned}
\mathcal{F}_{P}=\left\{\mathbf{F} \in \mathbb{R}^{3 M N \times N} \mid \mathbf{0} \preceq \operatorname{vec}(\mathbf{F}) \preceq \mathbf{1}_{3 M N},\right. \\
\left.\mathbf{F} \mathbf{1}_{N}=P \mathbf{1}_{3 M}\right\} .
\end{aligned}
$$

The correlation matrix of the demultiplexed images $\mathbf{R}$ can be obtained during the camera calibration phase; see Section VI-B. Concretely, we aim to solve the optimization problem

$$
\left\{\mathbf{F}^{\star}, \mathbf{G}^{\star}, P^{\star}\right\}=\arg \min _{\mathbf{F} \in \mathcal{F}_{P}, \mathbf{G} \in \mathbb{R}^{3 M \times N}, P \in \mathbb{R}} \mathcal{E}_{\mathrm{tc}}(\mathbf{F}, \mathbf{G}, P),
$$

By comparison to straightforward time and color multiplexing, i.e., $3 M=N$ and $\mathbf{F}=\mathbf{I}_{N}$, the gain of optimal time and color multiplexing is defined as

$$
G_{\mathrm{tc}}=\sqrt{\frac{\operatorname{Tr}\left(\boldsymbol{\Lambda}_{1}\right)}{\mathcal{E}_{\mathrm{tc}}\left(\mathbf{F}^{\star}, \mathbf{G}^{\star}, P^{\star}\right)}},
$$

where $\boldsymbol{\Lambda}_{1}$ is the noise covariance given by (17) with $P=1$.

The objective function of problem (19) is highly non-convex. Thus, directly handling this problem with a non-linear programming method is subject to the risk of converging to poor local optima, especially when the initial condition is given far away from the global optima. Some approximation and decomposition of problem (19) should be sought, with the hope that the solution found is less-sensitive to initial condition and that the subproblems can be convex and be easy to solve. In the following, we reformulate and approximate problem (21), shown at the bottom of the page, in such a way that the problem can be easily handled by sequential convex programming (SCP) and a 1-dimensional exhaustive search over a set of finite samples, where each subproblem involved in the SCP step is casted as a semi-definite programming (SDP) problem that can be readily solved by any convex optimization solver. We name the proposed method the Illumination Multiplexing Codes (IMC) algorithm.

\section{A. Illumination Multiplexing Codes (IMC) Algorithm}

Problem (21) is unconstrained with respect to $\mathbf{G}$, and thus we can first obtain a closed-form solution for $\mathbf{G}$, in terms of $\mathbf{F}$ and $P$, as follows:

$$
\mathbf{G}^{\star}=\left(\mathbf{F R F}^{T}+\boldsymbol{\Lambda}_{P}\right)^{-1} \mathbf{F R} .
$$

Substituting (23) into (21) yields

$$
\begin{aligned}
& \left\{\mathbf{F}^{\star}, P^{\star}\right\} \\
& \quad=\arg \min _{\mathbf{F} \in \mathcal{F}_{P}, P \in \mathbb{R}} \operatorname{Tr}\left(-\left(\mathbf{F R F}^{T}+\boldsymbol{\Lambda}_{P}\right)^{-1} \mathbf{F R}^{2} \mathbf{F}^{T}\right) .
\end{aligned}
$$

This problem, however, is still difficult to solve. To make the problem easier we introduce the following approximation

$$
\mathbf{R}^{2} \simeq \nu \mathbf{R} \text {, for some } \nu>0,
$$

where equality holds when all the eigenvalues of $\mathbf{R}$ are identical, or $\mathbf{s}[n]$ is statistically uncorrelated; i.e., $\mathbf{R}=\nu \mathbf{I}_{N}$. In our case, where $\mathbf{R}$ is the correlation matrix of the single-light source images $\mathbf{s}[n]$, one may arrange the light source directions to make $\mathbf{R}$ as close to $\nu \mathbf{I}_{N}$ as possible, thereby reducing the approximation error. A simple way is to adjust the directions of the illumination sources such that one illumination coverage region does not overlap much with the others; i.e., $\mathbb{E}\left(s_{i}[n] s_{j}[n]\right) \geq 0$ for $i \neq j$ is close to zero. One should also manage all the light sources such that the single-light source images $\mathbf{s}[n]$ have the same energy. This can be done by either varying the distances between the light sources and the object to be illuminated, or the power of light sources. As a rule of thumb, to make the approximation (25) tight, the light source directions should be arranged as disparate as possible, and their lighting energies should be roughly equal.

We now apply (25) to the objective function of problem (24) and get

$$
\begin{aligned}
\operatorname{Tr} & \left(-\alpha\left(\mathbf{F} \mathbf{R} \mathbf{F}^{T}+\boldsymbol{\Lambda}_{P}\right)^{-1} \mathbf{F R F}^{T}\right) \\
& =\operatorname{Tr}\left(-\alpha\left(\mathbf{F R F}^{T}+\boldsymbol{\Lambda}_{P}\right)^{-1}\left(\mathbf{F R F}^{T}+\boldsymbol{\Lambda}_{P}-\boldsymbol{\Lambda}_{P}\right)\right) \\
& =\operatorname{Tr}\left(\alpha\left(-\mathbf{I}_{3 M}+\left(\mathbf{F} \mathbf{R} \mathbf{F}^{T}+\boldsymbol{\Lambda}_{P}\right)^{-1} \boldsymbol{\Lambda}_{P}\right)\right) .
\end{aligned}
$$

After which, substituting (26) into (24) yields the approximation of problem (24):

$$
\min _{\mathbf{F} \in \mathcal{F}_{P}, P \in \mathbb{R}} \operatorname{Tr}\left(\left(\mathbf{F R F}^{T}+\boldsymbol{\Lambda}_{P}\right)^{-1} \boldsymbol{\Lambda}_{P}\right)
$$

Although problem (27) is already in a simplified form from (24) by using (23) and (25), optimizing it is still difficult due to the inverse operator in the objective function. Thus we adopt a divide-and-conquer strategy for problem (27) via SCP with $P$ fixed, and then we find the optimal $P$ through an exhaustive search. The strategy is described in the following two subsections.

1) Subproblem of (27) With P Fixed: If we suppose that the variable $P$ is fixed to the constant value $\hat{P}$, then problem (27) can be reformulated into the equivalent but alternative form:

$$
\min _{\mathbf{F} \in \mathcal{F}_{\hat{P}}, \mathbf{H} \in \mathbb{R}^{3 M \times 3 M}} \operatorname{Tr}\left(\mathbf{H} \boldsymbol{\Lambda}_{\hat{P}}\right), \quad \text { s.t. } \mathbf{H} \succeq\left(\mathbf{F R F} \mathbf{F}^{T}+\boldsymbol{\Lambda}_{\hat{P}}\right)^{-1},
$$

where the linear matrix inequality constraint can be further rewritten via Schur's complement ([17], Th. 7.7.6, p. 472) as

$$
\left[\begin{array}{cc}
\mathbf{H} & \mathbf{I}_{3 M} \\
\mathbf{I}_{3 M} & \mathbf{F R F}^{T}+\boldsymbol{\Lambda}_{\hat{P}}
\end{array}\right] \succeq \mathbf{0} .
$$

By letting

$$
\mathbf{U}=\mathbf{F R F}^{T},
$$


we can write problem (28) as

$$
\begin{array}{cl}
\min _{\mathbf{F} \in \mathcal{F}_{\hat{P}}, \mathbf{H}, \mathbf{U} \in \mathbb{R}^{3 M \times 3 M}} & \operatorname{Tr}\left(\mathbf{H} \boldsymbol{\Lambda}_{\hat{P}}\right) \\
\text { s.t. } & \mathbf{U}=\mathbf{F} \mathbf{R} \mathbf{F}^{T}, \\
& {\left[\begin{array}{cc}
\mathbf{H} & \mathbf{I}_{3 M} \\
\mathbf{I}_{3 M} & \mathbf{U}+\boldsymbol{\Lambda}_{\hat{P}}
\end{array}\right] \succeq \mathbf{0} .}
\end{array}
$$

Solving problem (31), however, is difficult because (31a) is nonconvex in $(\mathbf{F}, \mathbf{U})$. Thus we seek local optimization methods that approximate (31a) using a first-order Taylor series so as to make problem (31) solvable by a sequence of convex problems. Applying Newton's method [18] to the quadratic matrix equation

$$
Q(\mathbf{F})=\mathbf{U}-\mathbf{F R F}^{T}=\mathbf{0}
$$

where $Q: \mathbb{R}^{3 M \times 3 M} \mapsto \mathbb{R}^{3 M \times 3 M}$ is a continuously differentiable function, will have the following recurrence:

$$
\begin{gathered}
\text { given } \mathbf{F}_{0}(\text { initial guess of } \mathbf{F}), \\
\mathbf{U}-\mathbf{F}_{k} \mathbf{R} \mathbf{F}_{k}^{T}=\mathbf{F}_{k} \mathbf{R} \Delta \mathbf{F}^{T}+\Delta \mathbf{F} \mathbf{R} \mathbf{F}_{k}^{T}, \\
\mathbf{F}_{k+1}=\mathbf{F}_{k}+\Delta \mathbf{F}, \quad k=0,1,2, \ldots
\end{gathered}
$$

Here $\Delta \mathbf{F}$ denotes a small change in $\mathbf{F}$, and $\mathbf{F}_{k}$ denotes the estimate of $\mathbf{F}$ at iteration $k$. The newly updated $\mathbf{F}_{k+1}$ should be in the constraint set $\mathcal{F}_{\hat{P}}$. By replacing the equality constraint (31a) with (33b) at each iteration $k$, we can obtain the SDP formulation,

$$
\begin{aligned}
& \min _{\Delta \mathbf{F} \in \mathbb{R}^{3 M \times N}, \mathbf{H}, \mathbf{U} \in \mathbb{R}^{3 M \times 3 M}} \operatorname{Tr}\left(\mathbf{H} \boldsymbol{\Lambda}_{\hat{P}}\right) \\
& \text { s.t. } \mathbf{F}_{k}+\Delta \mathbf{F} \in \mathcal{F}_{\hat{P}}, \\
& \mathbf{U}-\mathbf{F}_{k} \mathbf{R} \mathbf{F}_{k}^{T}=\mathbf{F}_{k} \mathbf{R} \Delta \mathbf{F}^{T}+\Delta \mathbf{F} \mathbf{R} \mathbf{F}_{k}^{T}, \\
& \mathbf{H}, \mathbf{U} \text { satisfy }(31 \mathrm{~b}) .
\end{aligned}
$$

This SDP problem can be efficiently solved by any convex optimization solver [19]. Note that problem (31) is handled by a sequence of SDPs given by (34). In each iteration $k$, problem (34) is a local, linear approximation to problem (31). Once $\Delta \mathbf{F}$ is obtained at iteration $k$, we update $\mathbf{F}_{k+1}$ by (33c) and continue to solve problem (34) for $k:=k+1$. The complete SCP for problem (31) is outlined in Algorithm 1. How we give the initial $\mathbf{F}_{0}$ to Algorithm 1 is discussed in Remark 3 in Section IV. The iteration procedure will stop when the relative change in the objective function is smaller than a preset threshold.

2) Exhaustive Search Over Various P's: The remaining problem is to determine the optimal total energy of the activated light sources $P$. We apply a 1 -dimensional exhaustive search over finite samples of $P$, say $\hat{P}=2,3, \ldots, P_{\text {sat }}$, where we set $P_{\text {sat }}$ given by (14) as an upper bound to account for multiplexing saturation problems. After we obtain the optimal value $\bar{J}(\hat{P})$ for $\hat{P}=2,3, \ldots, P_{\text {sat }}$, a solution of $P$ and the time and color multiplexing matrix $\mathbf{F}$ to problem (24) can be found by

$$
\begin{aligned}
P^{\star} & =\min _{\hat{P}=2,3, \ldots, P_{\text {sat }}} \bar{J}(\hat{P}), \\
\mathbf{F}^{\star} & =\overline{\mathbf{F}}\left(P^{\star}\right) .
\end{aligned}
$$

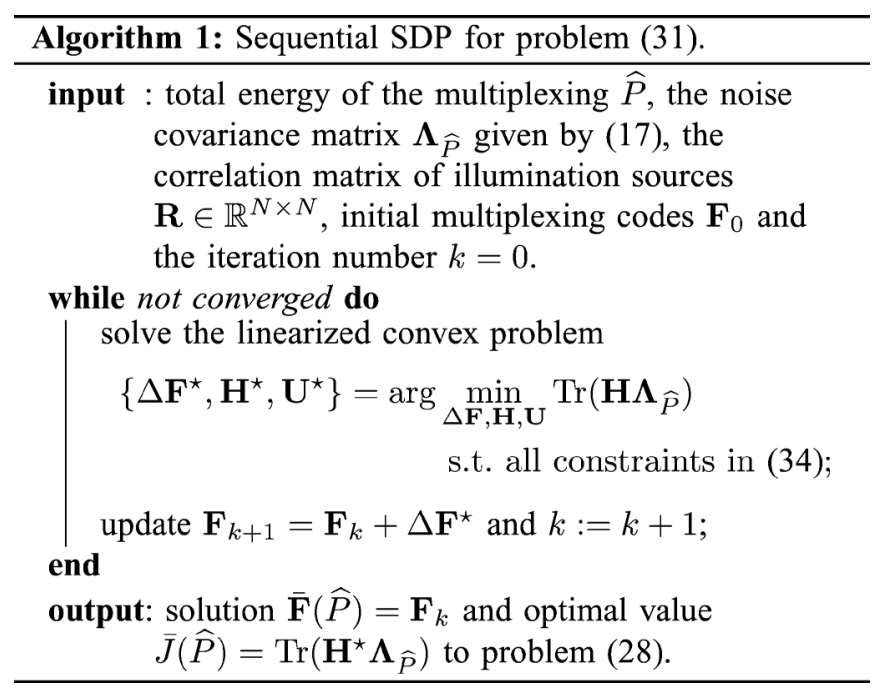

Finally, we summarize the IMC algorithm for problem (21) in Algorithm 2. The IMC algorithm can be readily applied to the MMSE design of time multiplexing codes only. The details are given in Appendix A.

\section{Algorithm 2: IMC algorithm for problem (21).}

input: total energy of the multiplexing saturation value $P_{\text {sat }}$ beyond which the captured images become saturated, the noise covariance matrix $\boldsymbol{\Lambda}_{\hat{P}}$ given by (17), and the correlation matrix of illumination sources $\mathbf{R} \in \mathbb{R}^{N \times N}$.

obtain $\overline{\mathbf{F}}(\hat{P})$ and $\bar{J}(\hat{P})$ by Algorithm 1 for $\hat{P}=2,3, \ldots, P_{\text {sat }}$. compute $P^{\star}, \mathbf{F}^{\star}$ and $\mathbf{G}^{\star}$ by (35), (36), and (23), respectively.

output: solution $P^{\star}, \mathbf{F}^{\star}$, and $\mathbf{G}^{\star}$ to problem (21).

3) Convergence Analysis of Algorithm 1: We now analyze the convergence of Algorithm 1 in this subsection. Unlike conventional sequential SDP [20] which applies Newton's method to solve the Karush-Kuhn-Tucker conditions of (31), the proposed Algorithm 1 employs Newton's method on the non-convex constraint $Q(\mathbf{F})=\mathbf{0}$ only. The local convergence for this type of algorithm has been analyzed in [21], whose results relate to the following nonconvex optimization problem,

$$
\min _{\mathbf{x} \in \mathbb{R}^{n}} \mathbf{c}^{T} \mathbf{x}, \quad \text { s.t. } g(\mathbf{x})=\mathbf{0}, \mathbf{x} \in \Omega
$$

where $\mathbf{c} \in \mathbb{R}^{n}, g(\mathbf{x}): \mathbb{R}^{d} \mapsto \mathbb{R}^{m}$ is non-linear and smooth on its domain, and $\Omega$ is a nonempty, closed, convex subset in $\mathbb{R}^{d}$. The authors of [21] studied the iterative method

$$
\begin{aligned}
\mathbf{x}_{k+1}=\arg \min _{\mathbf{x} \in \Omega} & \mathbf{c}^{T} \mathbf{x} \\
\text { s.t. } & g\left(\mathbf{x}_{k}\right)+\nabla g\left(\mathbf{x}_{k}\right)^{T}\left(\mathbf{x}-\mathbf{x}_{k}\right)=\mathbf{0},
\end{aligned}
$$


for $k=1,2, \ldots$ and showed that, under some contraction properties, if $\mathbf{x}^{\star}$ is a stationary point of (37) then within some neighborhood of $\mathbf{x}^{\star}$ the sequence generated by (38) converges linearly to $\mathrm{x}^{\star}$.

To connect our problem (31) to problem (37), we can define

$$
\begin{aligned}
\mathbf{x} & =h(\mathbf{F}, \mathbf{H}, \mathbf{U})=\left[\operatorname{vec}(\mathbf{F})^{T} \operatorname{vec}(\mathbf{H})^{T} \operatorname{vec}(\mathbf{U})^{T}\right]^{T}, \\
g(\mathbf{x}) & =g(h(\mathbf{F}, \mathbf{H}, \mathbf{U}))=\operatorname{vec}\left(\mathbf{U}-\mathbf{F R} \mathbf{F}^{T}\right)=\mathbf{0}, \\
\Omega & =\left\{\mathbf{x}=h(\mathbf{F}, \mathbf{H}, \mathbf{U}) \mid \mathbf{F} \in \mathcal{F}_{\hat{P}}, \mathbf{H}, \mathbf{U} \text { satisfy }(31 \mathrm{~b})\right\}, \\
\mathbf{c} & =\left[\mathbf{0}^{T} \mathbf{1}_{9 M M}^{T} \mathbf{0}^{T}\right]^{T},
\end{aligned}
$$

with $d=3 M N+18 M^{2}$ and $m=9 M^{2}$, and view the proposed method as a local procedure for solving problem (37) via the iterative process in (38). Hence, the results of [21] suggest that our method solves a sequence of problems (34) and converges linearly to a stationary point of problem (31).

\section{IMC-Like AlgORIthm For MAX-SNR BASEd TIME AND COLOR MULTIPLEXING}

This section presents the application of an IMC-like algorithm to compute the max-SNR based time and color multiplexing codes. The algorithm's development basically follows the key steps in Section III. As has been discussed previously, the max-SNR design is thought of as the MMSE code design problem (21) with an extra constraint $\mathbf{G}^{T} \mathbf{F}=\mathbf{I}_{N}$ :

$$
\begin{array}{r}
\left\{\mathbf{F}^{\sharp}, \mathbf{G}^{\sharp}, P^{\sharp}\right\}=\arg \min _{\mathbf{F} \in \mathcal{F}_{P}, \mathbf{G} \in \mathbb{R}^{3 M} \times N, P \in \mathbb{R}} \operatorname{Tr}\left(\mathbf{G} \boldsymbol{\Lambda}_{P} \mathbf{G}^{T}\right) \\
\text { s.t. } \quad \mathbf{G}^{T} \mathbf{F}=\mathbf{I}_{N} .
\end{array}
$$

Note that the above max-SNR design (39) does not hinge on the illumination correlation matrix $\mathbf{R}$, therefore no approximation like the one performed in (25) for MMSE design is needed. To make the IMC algorithm applicable to handling (39), we now reformulate it as follows. By the Lagrange multiplier method [22], we can obtain a closed-form solution of $\mathbf{G}$ :

$$
\mathbf{G}^{\sharp}=\boldsymbol{\Lambda}_{P}^{-1} \mathbf{F}\left(\mathbf{F}^{T} \boldsymbol{\Lambda}_{P}^{-1} \mathbf{F}\right)^{-1} .
$$

The details of how we derive (40) can be found in the Appendix B. Substituting (40) into (39) yields

$$
\left\{\mathbf{F}^{\sharp}, P^{\sharp}\right\}=\arg \min _{\mathbf{F} \in \mathcal{F}_{P}, P \in \mathbb{R}} \operatorname{Tr}\left(\left(\mathbf{F}^{T} \boldsymbol{\Lambda}_{P}^{-1} \mathbf{F}\right)^{-1}\right) .
$$

Compared to standard time and color multiplexing, i.e., $3 M=$ $N$ and $\mathbf{F}=\mathbf{I}_{N}$, the gain of time and color multiplexing associated with the max-SNR design is then computed as

$$
G_{\mathrm{tc}}=\sqrt{\frac{\operatorname{Tr}\left(\boldsymbol{\Lambda}_{1}\right)}{\operatorname{Tr}\left(\left(\left(\mathbf{F}^{\sharp}\right)^{T} \boldsymbol{\Lambda}_{P^{\sharp}}^{-1} \mathbf{F}^{\sharp}\right)^{-1}\right)}} .
$$

Now we are faced with the question of solving problem (41). Using tools similar to those presented in Section III.A, such as

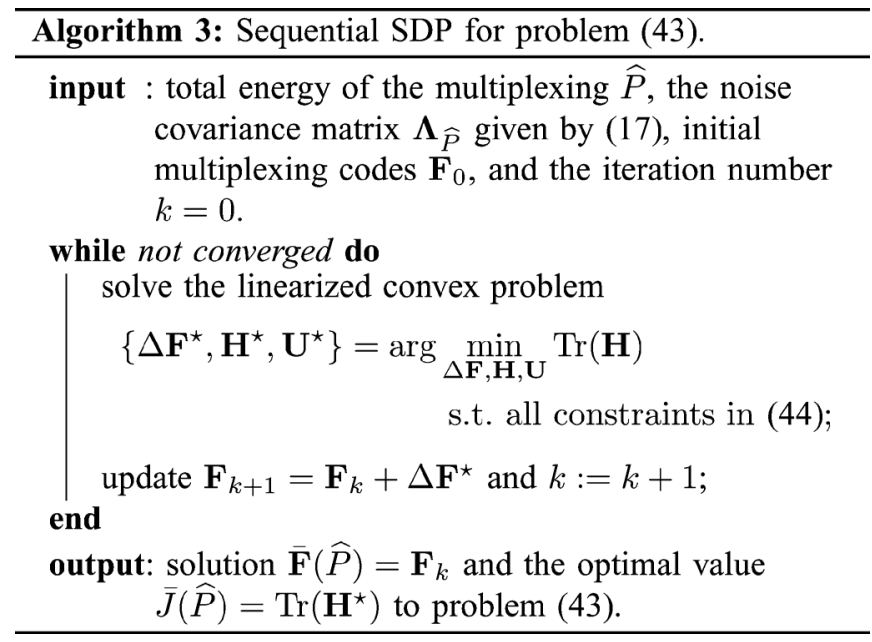

TABLE I

COMPARISON BETWEEN MMSE AND MAX-SNR DESIGNS

\begin{tabular}{c|c|c|c|c}
\hline Designs & Multi. gain & Efficiency & Approx. & Given info. \\
\hline \hline MMSE & higher & lower & Eqs. (25), (31a) & $\boldsymbol{\Lambda}_{P}, \mathbf{R}$ \\
max-SNR & lower & higher & Eq. (43a) & $\boldsymbol{\Lambda}_{P}$ \\
\hline
\end{tabular}

the divide-and-conquer strategy and Schur's complement, one can obtain via $P$ fixed to $\hat{P}$

$$
\begin{array}{cl}
\min _{\mathbf{F} \in \mathcal{F}_{\hat{P}}, \mathbf{H}, \mathbf{U} \in \mathbb{R}^{N \times N}} & \operatorname{Tr}(\mathbf{H}) \\
\text { s.t. } & \mathbf{U}=\mathbf{F}^{T} \boldsymbol{\Lambda}_{\hat{P}}^{-1} \mathbf{F}, \\
& {\left[\begin{array}{cc}
\mathbf{H} & \mathbf{I}_{N} \\
\mathbf{I}_{N} & \mathbf{U}
\end{array}\right] \succeq \mathbf{0 .} .}
\end{array}
$$

Again, (43a) is non-convex. Hence, we can apply Newton's method [18] to the constraint, thus granting us the following sequential SDP formulation:

$$
\begin{aligned}
& \min _{\Delta \mathbf{F} \in \mathbb{R}^{3 M \times N}, \mathbf{H}, \mathbf{U} \in \mathbb{R}^{N \times N}} \operatorname{Tr}(\mathbf{H}) \\
& \text { s.t. } \quad \mathbf{F}_{k}+\Delta \mathbf{F} \in \mathcal{F}_{\hat{P}} \\
& \quad \mathbf{U}-\mathbf{F}_{k}^{T} \boldsymbol{\Lambda}_{\hat{P}}^{-1} \mathbf{F}_{k}=\mathbf{F}_{k}^{T} \boldsymbol{\Lambda}_{\hat{P}}^{-1} \Delta \mathbf{F}+\Delta \mathbf{F}^{T} \boldsymbol{\Lambda}_{\hat{P}}^{-1} \mathbf{F}_{k}, \\
& \quad \mathbf{H}, \mathbf{U} \text { satisfy (43b), }
\end{aligned}
$$

where $k$ is the current number of iterations. Putting the above problem into an iterative SCP framework, we have the pseudocode of SCP for problem (43) in Algorithm 3. To do this, the initial $\mathbf{F}_{0}$ should be feasible; thus the elements of $\mathbf{F}_{0}$ are randomly generated following a uniform distribution over $[0,1]$, and they are normalized to fall in $\mathcal{F}_{\hat{P}}$. After applying Algorithm 3 to the problem with various $\hat{P}$ 's, the solution terms $P^{\sharp}$ and $\mathbf{F}^{\sharp}$ can be found by (35) and (36), respectively. Both the detailed version of the max-SNR design based time and color multiplexing codes along with its application to time multiplexing can be found in the short version of this paper [15].

We conclude this section with the following remarks, along with Table I to summarize the pros and cons of the MMSE design and max-SNR design. 
Remark 1: The MMSE-based formulation and algorithm are more general than those of the max-SNR design, and it has been shown in our derivation that the former provides a multiplexing gain no less than the latter. However, at each SCP iteration, the MMSE design needs to solve problem (34) with $3 M N+18 M^{2}$ variables, while the max-SNR design tackles problem (44) with $3 M N+2 N^{2}$ unknowns. Since $3 M \geq N$, we can infer that the MMSE design problem size is bounded from below by the problem size of the max-SNR design. Accordingly, computing the multiplexing codes of the max-SNR design is more efficient than doing so for the MMSE design, especially as the number of multiplexed illuminations $M$ increases.

Remark 2: Compared to the max-SNR design which only needs the noise covariance matrix $\boldsymbol{\Lambda}_{P}$ for computing the multiplexing codes, the extra information we need for the MMSE design is the correlation matrix of illumination sources $\mathbf{R}$. Moreover, MMSE also needs the approximation (25), which can be tight if the illumination sources are diverse enough, or the illumination sources tend to be uncorrelated. As we will see in simulations, even though there is some approximation error in (25), the MMSE multiplexing codes still yield a multiplexing gain larger than that of the max-SNR design.

Moreover, we discuss the algorithm initialization in the following remark.

Remark 3: Since the computational load of the MMSE design is heavier than that of the max-SNR design, and the feasible set of the max-SNR design is a subset of that of the MMSE design, it is natural to use a max-SNR solution to initialize Algorithm 1. This strategy can save a large amount of the computation time of the MMSE debigrCOMPUTER SIMULATIONS

The multiplexing gain performance of the proposed IMC algorithms based on the MMSE and max-SNR criteria will be demonstrated for both time multiplexing and time and color multiplexing in this section. To ease the ensuing presentation, we label the proposed IMC algorithm for MMSE design as IMC-MMSE, and we label that for the max-SNR design as IMC-mSNR.

In the following simulation we used a pink piglet as the illuminated object; see Fig. 2(a). We then calculated the material color of the object as required by time and color multiplexing. Suppose that the RGB values of the acquired color image are $\left\{z_{r}[n]\right\}_{n=1}^{L},\left\{z_{g}[n]\right\}_{n=1}^{L},\left\{z_{b}[n]\right\}_{n=1}^{L}$. The material colors can then be calculated by $\alpha_{c}[n]=z_{c}[n] /\left(z_{r}[n]+z_{g}[n]+z_{b}[n]\right), n=$ $1, \ldots, L, c \in\{r, g, b\}$. Fig. 2(b) shows the synthetic map of the material colors. In addition, we collected $N=12$ single-light source illuminations as the ground-truth $\mathbf{s}[n]$, as displayed in Fig. 2(c). Under these illumination settings, the approximation error in (25) is $\min _{\alpha>0}\left\|\mathbf{R}^{2}-\alpha \mathbf{R}\right\|_{F} /\left\|\mathbf{R}^{2}\right\|_{F}=0.0131$. Note that the correlation matrix of illumination sources $\mathbf{R}$ (to be used in IMC-MMSE) is now assumed to be known; exactly how we practically estimate $\mathbf{R}$ in real experiments will be detailed in Section VI-A.

\section{A. Time Multiplexing}

We show results with time multiplexing in order to make a fair comparison with Ratner's method [10], a state-of-the-art method based on max-SNR design used for time multiplexing. There are $M=12$ or 24 measurements allowed to be captured
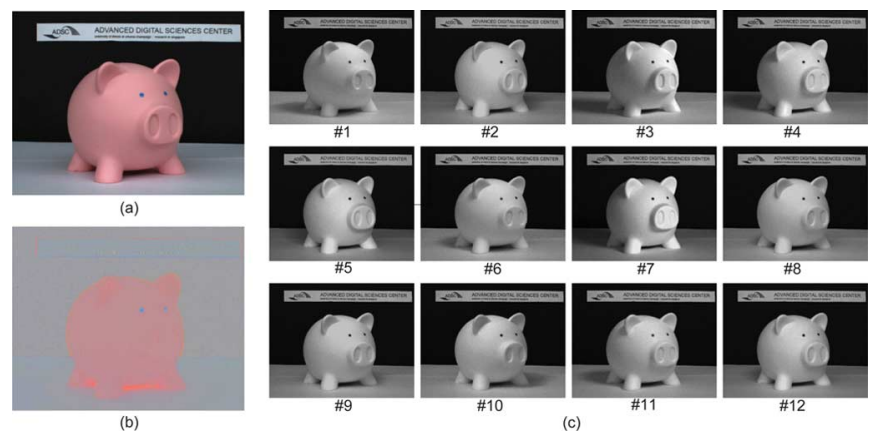

Fig. 2. (a) The illuminated object, (b) the calculated material colors of the object, and (c) the twelve single-light source illuminations.

by using time multiplexing. The two noise parameters given by (3) are set to $\sigma^{2}=1.5$ and $\rho^{2}=0.015 \sigma^{2}$. Fig. 3(a) shows the multiplexing gain performance of the proposed IMC-MMSE and IMC-mSNR algorithms, as well as the Ratner's method [10], for various $P$ 's. One can see that IMC-MMSE achieves a significantly higher multiplexing gain than the max-SNR based methods; i.e., IMC-mSNR and Ratner's method, for both $M=$ 12 and $M=24$. IMC-mSNR outperforms Ratner's method ${ }^{5}$ for $M=24$ and achieves comparable performance with Ratner's method for $M=12$. Fig. 4(a) shows the time multiplexing codes yielded by the IMC-MMSE method for $P^{\star}=6$ and $M=24$. We see that the time multiplexing codes are rather diverse. Moreover, the performance improvement can be seen obviously as the number of measurements $M$ increases, regardless of the value of $P$. All the above observations immediately suggest that if someone demands a high-quality image under a single light source condition, the solution is to acquire more measurements using the MMSE-based multiplexing codes. The proposed IMC-MMSE method offers such multiplexing codes!

\section{B. Time and Color Multiplexing}

We now consider time and color multiplexing where there are $M=4$ and 8 RGB multiplexed images. Although we use a small number of measurements than the time multiplexing in the last subsection, $M=4$ or 8 RGB multiplexed images are equivalent to $3 M=12$ or 24 monochrome multiplexed images, respectively. The RGB noise parameters $\Lambda_{P}$ given by (17) are set to $\sigma_{r}^{2}=1, \sigma_{g}^{2}=0.9 \sigma_{r}^{2}, \sigma_{b}^{2}=2 \sigma_{r}^{2}$, and $\rho_{c}^{2}=\chi^{2} \sigma_{c}^{2}, c \in$ $\{r, g, b\}$ where $\chi^{2}$ denotes the common ratio of the sensor noise power and photon noise power for the RGB channels. For noise correlation across RGB channels, we use an affine function ${ }^{6}$ of $P$; i.e., $\boldsymbol{\Theta}_{P}^{i j}=\sigma_{x}^{2}+P \rho_{x}^{2}$ for all $i, j \in\{r, g, b\}$, with $\sigma_{x}^{2}=0.013$ and $\rho_{x}^{2}=-\chi^{2} \sigma_{x}^{2}$

Fig. 3(b) shows the time and color multiplexing gain of the proposed IMC-MMSE and IMC-mSNR methods for $\chi^{2}=0.015$ and various $P$ 's. It can be observed that the multiplexing gains of both methods improve as the number of measurements $M$ increases, and IMC-MMSE achieves a much

\footnotetext{
${ }^{5}$ Ratner's method was developed for the determined multiplexing system $M=N$. For the case of $M=24$, we concatenate the same Ratner multiplexing codes of $M=12$ twice to serve as the multiplexing matrix, and we use this for a baseline comparison.

${ }^{6}$ This kind of affine function applies to the real scenarios in our experiments; see Section VI.
} 


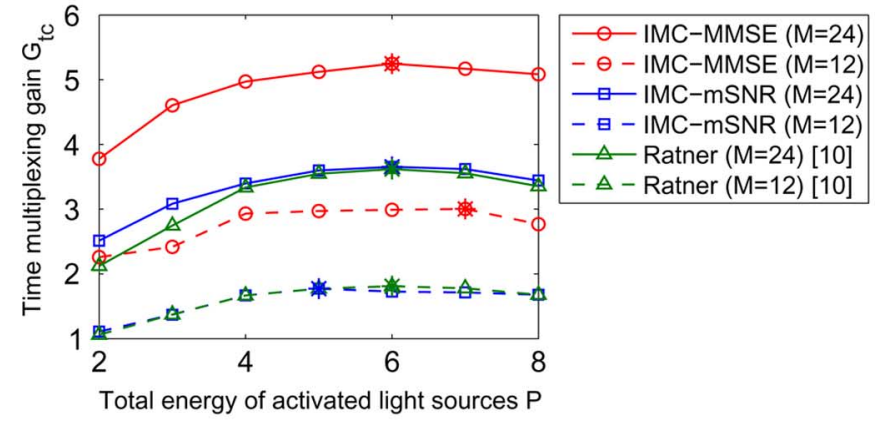

(a)

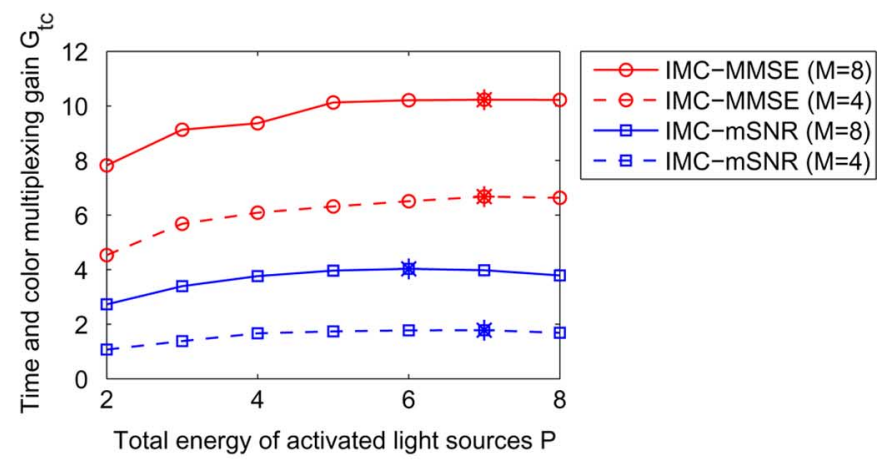

(b)

Fig. 3. (a) Time multiplexing for $M=12$ and 24 over various $P$ 's. (b) Time and color multiplexing for $M=4$ and 8 over various $P$ 's. The symbol ' $*$ ' denotes the maximum multiplexing gain for a specific setting, and this informs us of the optimal $P$.

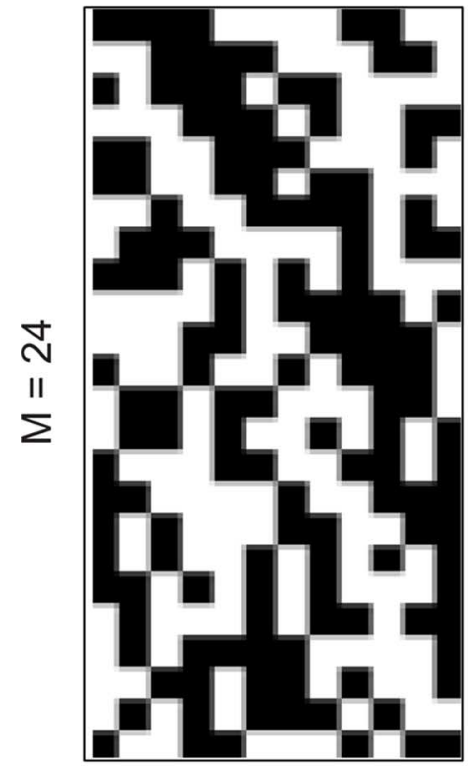

$\mathrm{N}$

(a)

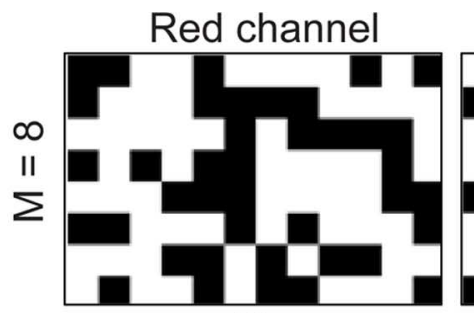

$\mathrm{N}$

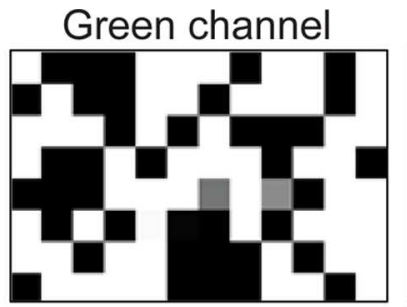

$\mathrm{N}$<smiles>C[12CH2]</smiles>
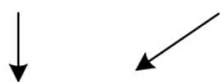

RGB composed

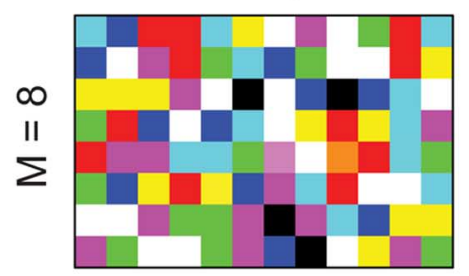

$\mathrm{N}$

(b)

Fig. 4. (a) Time multiplexing codes yielded by the proposed IMC-MMSE for $P^{\star}=6$ and $M=24$. (b) Time and color multiplexing codes yielded by the proposed IMC-MMSE for $P^{\star}=7$ and $M=8$. [Top] Shown from the left to the right are the time multiplexing codes $\mathbf{F}_{r}, \mathbf{F}_{g}, \mathbf{F}_{b}$ for the RGB channels, respectively; [Bottom] the composed RGB multiplexing patterns. For the time multiplexing codes of any given color channel, fully bright segments mean the codes take a value of 1 (specifying full illumination), whereas fully dark segments indicate a coded value of 0 (specifying no illumination).

higher multiplexing gain than IMC-mSNR. Fig. 4(b) displays the time and color multiplexing codes and the composed RGB multiplexing patterns yielded by the IMC-MMSE method for $P^{\star}=7$ and $M=8$. One can see that the time multiplexing codes for each RGB channel are diverse, and the composed RGB multiplexing patterns are not limited to some specific colors.

To make more sense of how multiplexing really improves image quality, we synthetically generate the multiplexed images by following (16) with the calculated multiplexing matrix of $P^{\star}$, and we subsequently de-multiplex them to infer the illumination sources. Fig. 5 displays the de-multiplexed images of IMC-MMSE, IMC-mSNR and the conventional illuminations (i.e., $\mathbf{F}=\mathbf{I}_{M}$ and $M=N=12$ ). One can clearly see that the quality of the images demultiplexed by IMC-MMSE and IMC-
mSNR is greatly enhanced compared to the conventional illuminations, with IMC-MMSE making the largest improvement.

\section{EXPERIMENTS}

We use object illumination to demonstrate the effectiveness of the proposed time and color multiplexing methods, IMC-MMSE and IMC-mSNR. A sketch of the illumination multiplexing system is shown in Fig. 6(a). Two PC-controlled BENQ MX761 projectors create colored illumination patterns of $N=24$ lit segments, shown in Fig. 6(b), on two diffuse-white walls straddling the corner of a room. Light reflected by these lit patches acted as separate light sources illuminating the placed object. Two kinds of objects were considered: Lambertian objects and shiny (non-Lambertian) objects; see Fig. 7. While the illumination superposition model 
(a)

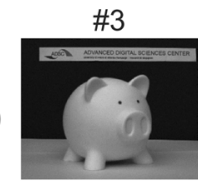

(b)

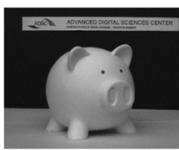

(c)

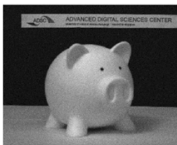

(d)

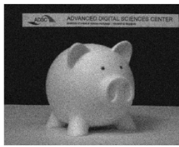

(e)
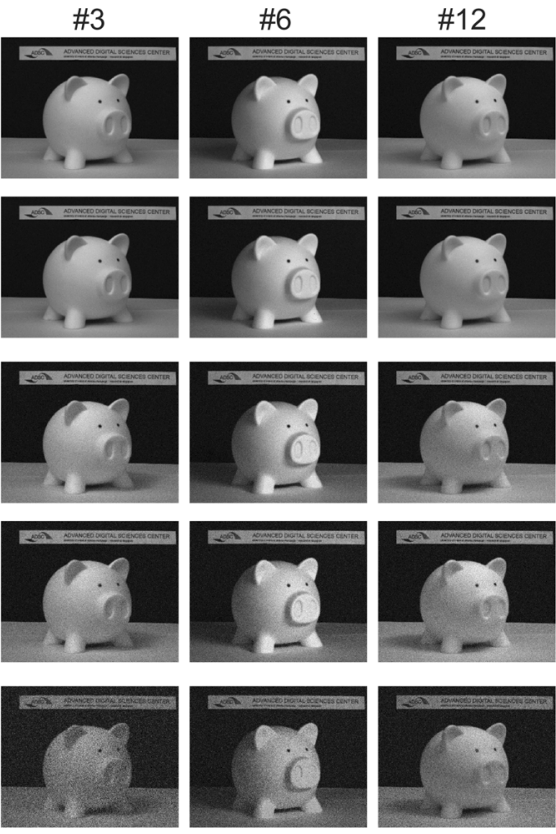

Fig. 5. Three time and color demultiplexed images (\#3,\#6,\#12) using the matrix of the optimal $P^{\star}$ obtained by (a) IMC-MMSE $(M=8)$, (b) IMC-MMSE $(M=4)$, (c) IMC-mSNR $(M=8)$, (d) IMC-mSNR $(M=4)$. (e) The conventional illuminations; i.e., $\mathbf{F}=\mathbf{I}_{M}$ with $M=N=12$

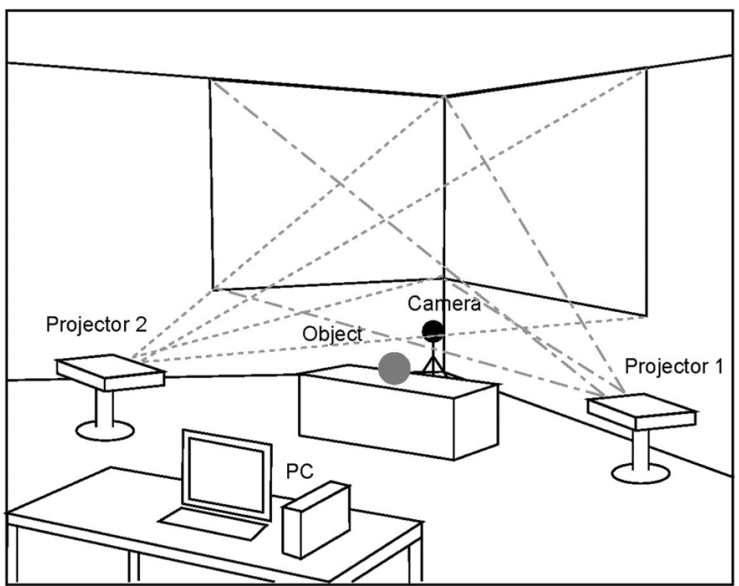

(a)

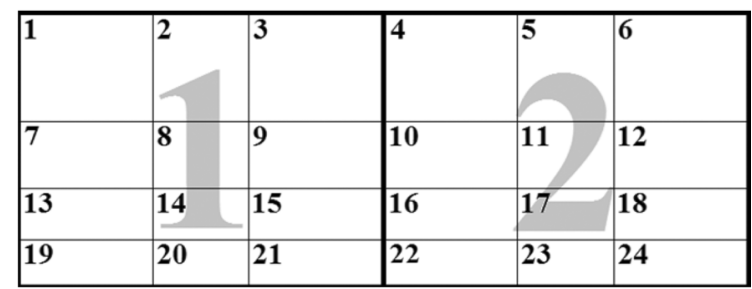

(b)

Fig. 6. (a) Experimental setup, and (b) the illumination patterns.

(15) should hold only for Lambertian objects, use of the shiny objects helps to evaluate the limits of the proposed methods. A FUJINON FL2G13S2C-C camera was used to capture the multiplexed images, and its exposure time and amplifier gain were set to $100 \mathrm{msec}$ and $0 \mathrm{~dB}$, respectively.

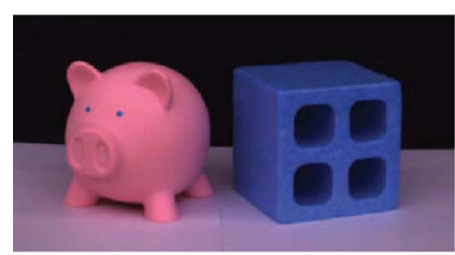

(a)

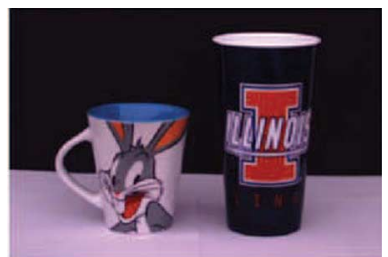

(b)
Fig. 7. The objects used in the experiments. (a) Lambertian objects, and (b) the shiny (non-Lambertian) objects.

\section{A. Projector Calibration}

Two issues must be considered during the camera calibration. The first is the nonlinear response of the camera readout as the intensity of the light source increases linearly. The usual step for fixing this behavior is to enable $\gamma$-correction mode in the camera, but this is not advised for multiplexing [4]. Instead, we compensate this nonlinearity by assigning nonlinear reference values to the hard-coded light source intensities such that the illuminations measured by the camera are linear in light intensity. Specifically, we use the nonlinear function, $f(x)=\eta x^{1 / \gamma}$ where $\eta=0.98$ and $\gamma=2$, to transform the linear input $x \in[0,1]$ to a reference value $f(x)$ for the projector to display the appropriate strength of lighting. The second issue is the spectral overlap among the camera's RGB channels. Since the spectral bands of the red and blue channels are almost disjoint whereas the green channel is overlapped with both the red and blue channels, we therefore only use the red and blue channels for time and color multiplexing.

\section{B. Estimation of Noise Covariance and Single-Light Source Covariance}

When designing the optimal time and color multiplexing codes, the measurement noise variances $\sigma_{r}^{2}, \sigma_{b}^{2}$ and its cross-correlation $\sigma_{r b}^{2}, \sigma_{b r}^{2}$, as well as photon noise variances $\rho_{r}^{2}, \rho_{b}^{2}$ and its cross-correlation $\rho_{r b}^{2}, \rho_{b r}^{2}$ play a central role. We follow the method reported in [10] to estimate the variances of the photon noise and sensor noise for the red and blue channels of the camera, and the measured noise variances as a function of $P$ is shown in Fig. 8. It is clear that the measured noise variances are an affine function of $P$. Using a simple line fitting, the resultant variances of the sensor noise are $\sigma_{r}^{2}=1.1567$ and $\sigma_{b}^{2}=1.1942$, while the resultant variances of the photon noise are $\rho_{r}^{2}=0.5052$ and $\rho_{b}^{2}=0.5716$. In addition, the parameters of the noise correlation between the red and blue channels are $\sigma_{r b}^{2}=\sigma_{r b}^{2}=0.0301$ and $\rho_{r b}^{2}=\rho_{r b}^{2}=-0.0104$.

A pre-scan of the object under white light conditions is required in order to estimate the RGB material colors of the object. The synthetic map of the material colors is shown in Fig. 9. With all the noise parameters and the material colors, we can easily compute from (17) the covariance matrix $\boldsymbol{\Lambda}_{P}$ for $P=1$. Due to the statistical independence between the illumination sources and the noise, the correlation matrix of illumination sources is then computed by $\mathbf{R}=\mathbb{E}\left(\mathbf{y}[n] \mathbf{y}[n]^{T}\right)-\boldsymbol{\Lambda}_{1}$, where $\mathbf{y}[n]$ is the conventional multiplexed measurements (16) with $\mathbf{F}=\mathbf{I}_{M}$ and $M=N$, after re-weighted by the material colors. 


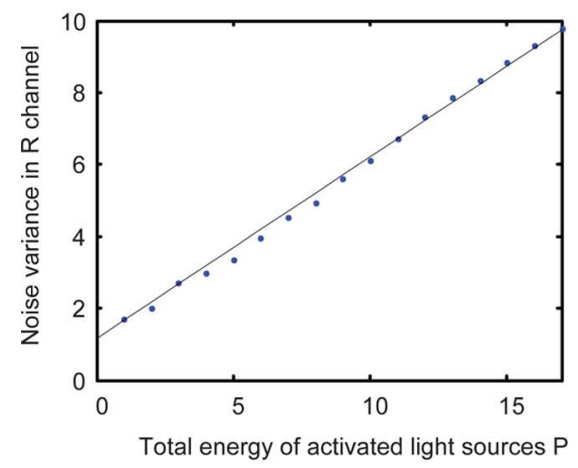

(a)

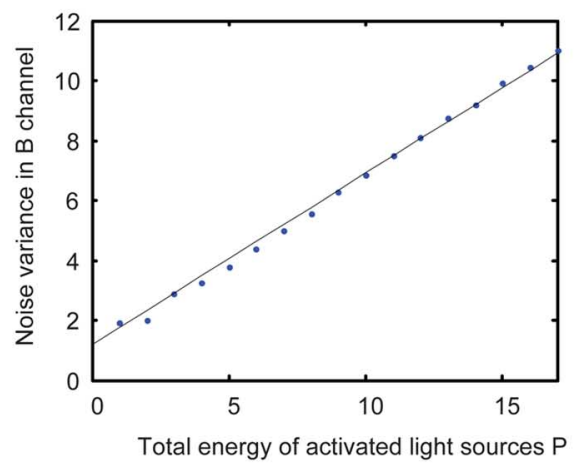

(b)

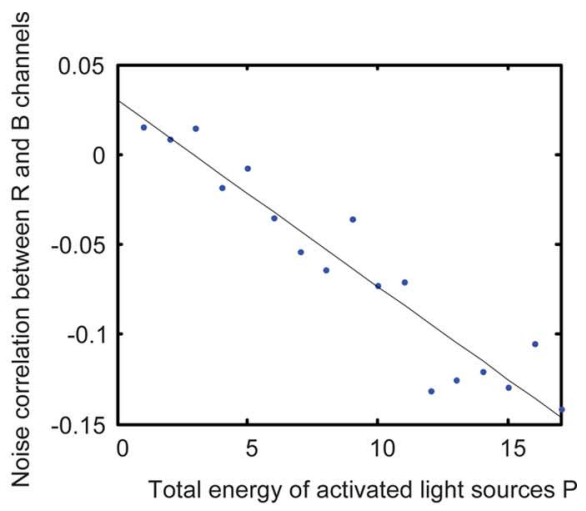

(c)

Fig. 8. Noise calibration as a function of $P$. (a) Noise variance in the red channel, and (b) in the blue channel. (c) Noise correlation between the red and blue channels.

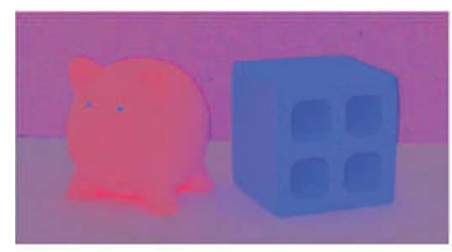

(a)

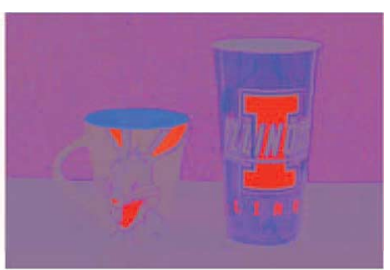

(b)
Fig. 9. The synthetic map of the objects' calculated material colors. (a) Lambertian objects, and (b) shiny (non-Lambertian) objects.

\section{Object Illuminations}

Just as the noise variances, covariance of single-source illuminations, and the material colors were acquired in the above subsections, the time and color multiplexing codes, either based on MMSE or max-SNR, can be easily computed by using the proposed methods. We gathered $M=12$ and 24 time and color multiplexed measurements for the objects used in the experiment, and performed demultiplexing from the $M$ multiplexed images. Since the demultiplexed single-light source illuminations $\{\hat{\mathbf{s}}[n]\}_{n=1}^{L}$ do not contain any color information, we incorporate the material colors into $\{\hat{\mathbf{s}}[n]\}_{n=1}^{L}$ to obtain the RGB single-light source illuminations, where the intensity of the $j$ th illumination in the RGB channels are written as $\left\{\alpha_{c}[n] \hat{s}_{j}[n]\right\}_{n=1}^{L}, c \in\{r, g, b\}$. The results of different objects are presented in the following subsections.

1) Lambertian Objects: The Lambertian objects of this experiment are a pink piglet and a blue styrofoam brick with four holes. Fig. 10(a) shows the time and color multiplexing gain $G_{\mathrm{tc}}$ for the MMSE-based and max-SNR based multiplexed images with $M=12$ and 24. In this experimental setting, IMC-MMSE yields a greater multiplexing gain than IMC-mSNR for any given $M$, and using $M=24$ multiplexed images more than doubled the multiplexing gain improvement in comparison to using $M=12$. Fig. 12 shows the 14 th and 17 th RGB single-light source illuminations obtained from the $M=12$ and 24 time and color multiplexed images and from the trivial illumination $M=12, \mathbf{F}=\mathbf{I}_{24}$, respectively. One can clearly see that the quality of the MMSE-based demultiplexed images, in terms of their visual appearance, is better than that of the max-SNR based demultiplexed images, and the quality of these demultiplexed images improve as the number of time and color multiplexing measurements $M$ increases. Also noticeable are some artifacts around the edges of the holes in the blue styrofoam object in the max-SNR based demultiplexed images [Fig. 12(c)-(d)], whereas the MMSE-based demultiplexed images do not have such a phenomenon. For a quantitative analysis, we computed the variances of the signals within a $100 \times 100$ pixel square in the black background of these demultiplexed images. These values, referred to as the noise variance, were calculated to be $0.4575,0.5416,0.7128$, 1.0098 , and 1.7221 for IMC-MMSE $(M=24)$, IMC-MMSE $(M=12), \operatorname{IMC}-m S N R(M=24), \operatorname{IMC}-m S N R(M=12)$, and the conventional illumination, respectively. This shows the benefits of using MMSE-based time and color multiplexing as well as a greater number of measurements.

2) Non-Lambertian Objects: Now, we tackle a more challenging case, illuminating shiny cups for which the illumination superposition model (15) may not be satisfied. Fig. 10(b) shows the time and color multiplexing gain $G_{\mathrm{tc}}$ for the MMSE-based and max-SNR based multiplexed images, with $M=12$ and 24 . The observation is similar to the Lambertian objects: the multiplexing gain improves when the number of multiplexed images $M$ increases, but the performance gap between the MMSE design and the max-SNR design is larger than that of the Lambertian objects [Fig. 10(a)]. Fig. 12 shows the 3rd and 23rd RGB single-light source illuminations obtained from both the $M=12$ and 24 time and color multiplexed images and from the trivial illumination $M=12, \mathbf{F}=\mathbf{I}_{24}$, respectively. Visually one observes that the quality of the MMSE-based demultiplexed images is higher than that of the max-SNR based demultiplexed images, though both types improve the quality as $M$ increases. Moreover, we computed the noise variance of these demultiplexed images for a qualitative comparison, and they are 0.0709, $0.0999,0.4416,0.6624$, and 1.2321 for IMC-MMSE $(M=24)$, IMC-MMSE $(M=12)$, IMC-mSNR $(M=24)$, IMC-mSNR $(M=12)$, and the conventional illumination, respectively. This again confirms the merits of using an MMSE-based time and color multiplexing, along with a greater number of measurements. 


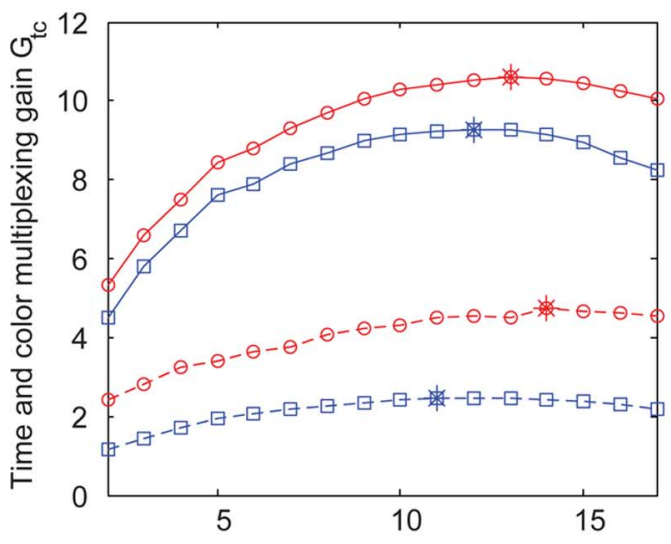

Total energy of activated light sources $\mathrm{P}$

(a)

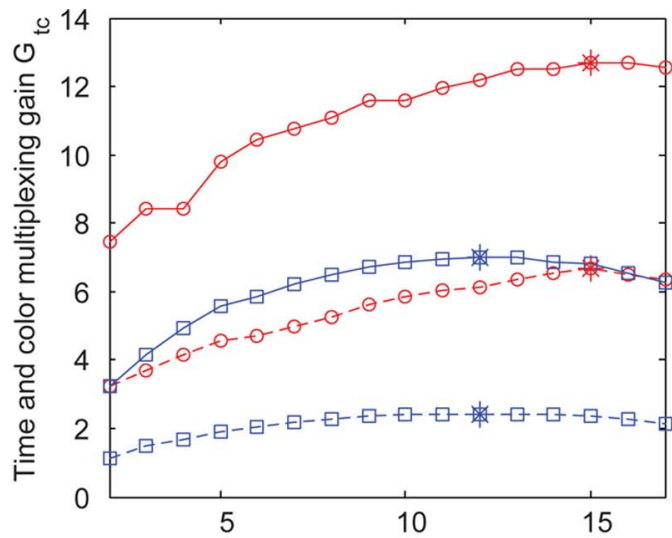

Total energy of activated light sources $P$

Fig. 10. Time and color multiplexing for $M=12$ and 24 over various $P$ 's. (a) Lambertian objects. (b) shiny (non-Lambertian) objects. The symbol ' $*$ ' denotes the maximum multiplexing gain for a specific setting, and this informs us of the optimal $P^{\star}$

\#14

(a)

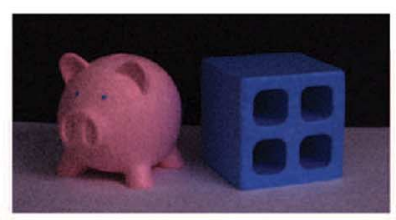

(b)

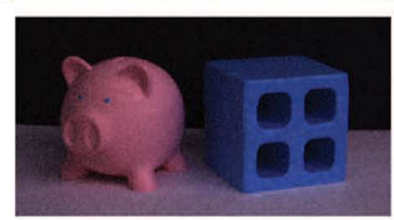

(c)

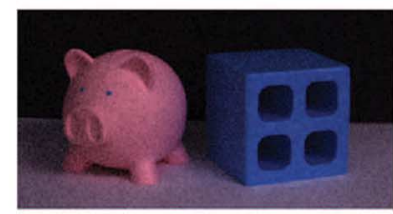

(d)

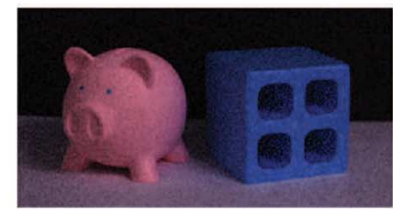

(e)

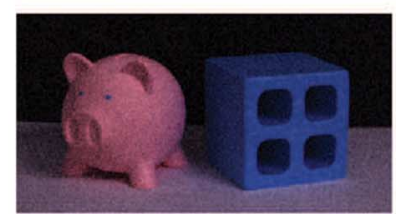

\#17
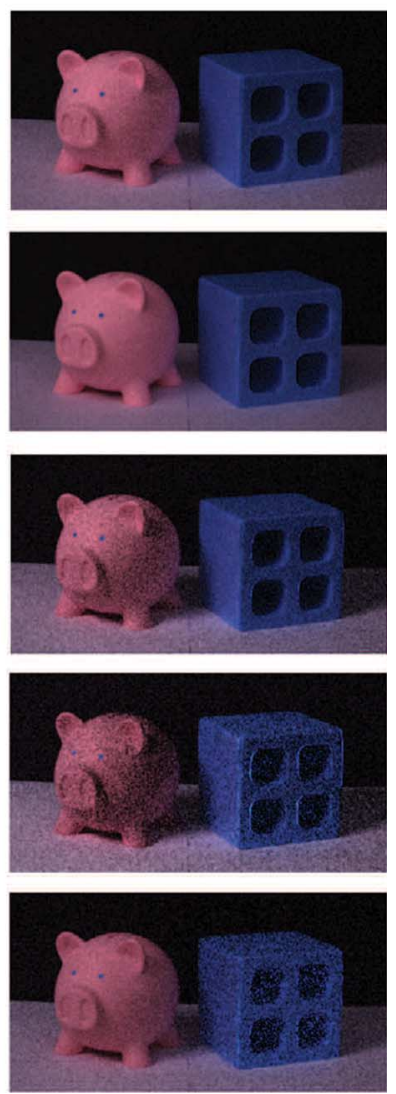

Fig. 11. Two of the time and color demultiplexed images (\#14, \#17) using the matrix of $P^{\star}$ obtained by (a) IMC-MMSE $(M=24)$, (b) IMC-MMSE $(M=$ $12)$, (c) IMC-mSNR $(M=24)$, (d) IMC-mSNR $(M=12)$. (e) Conventional illuminations; i.e., $\mathbf{F}=\mathbf{I}_{M}$ and $M=N=12$.

\section{CONCLUSION}

We have formulated an MMSE code design problem for time and color multiplexing with $K M \geq N$, which can be thought of as a generalization of the conventional max-SNR code design problem. We have handled the formulated MMSE problem by proposing the IMC algorithm, which includes two main components: iterative SCP and a 1-dimensional exhaustive search.

\#3

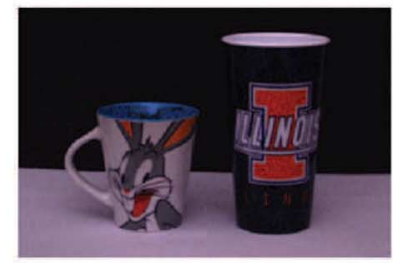

(b)

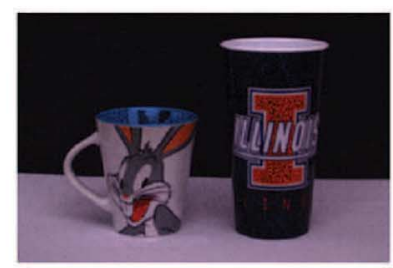

(c)

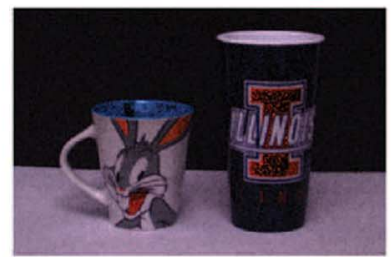

(d)

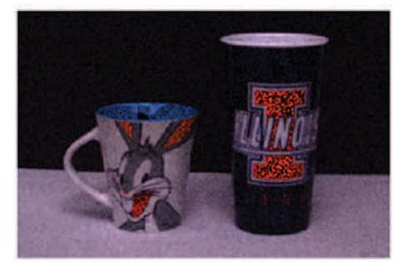

(e)
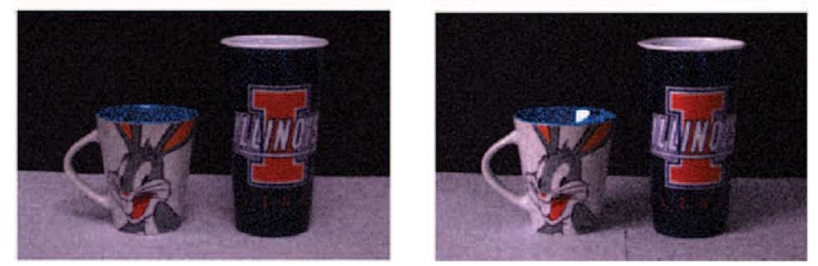

Fig. 12. Two of the time and color demultiplexed images (\#3, \#23) using the matrix of the optimal $P$ obtained by (a) IMC-MMSE $(M=24)$, (b) IMCMMSE $(M=12)$, (c) IMC-mSNR $(M=24)$, (d) IMC-mSNR $(M=12)$. (e) Conventional illuminations; i.e., $\mathbf{F}=\mathbf{I}_{M}$ and $M=N=12$.

Not only can the proposed IMC algorithm compute the MMSE based time and color multiplexing codes, but it can also be 
applied to MMSE-based time multiplexing, max-SNR based time multiplexing, and both time and color multiplexing. We anticipate that it will open a new door for a general setting (without the limitation of $M=N$ ) in MMSE-based, max-SNR based, or time and color multiplexing. As deduced from the simulations and experimental results, we summarize the primary merits of the proposed formulations and methods into the following points:

- The MMSE-based method outperforms the max-SNR method in terms of multiplexing gain.

- The multiplexing gains of MMSE-based and max-SNR based methods significantly increase as $M$ increases.

- Only $1 / K$ measurements are required in time and color multiplexing for achieving similar or even better performance to the case when only time multiplexing is used.

- The above advantages not only hold true for Lambertian objects, but also for non-Lambertian objects.

These unique results are hardly discussed in the existing works [1], [2], [4], [9], [10].

\section{APPENDIX}

\section{A. IMC Algorithm for MMSE Based Time Multiplexing}

The methodology developed above can also be applied to time multiplexing. Recall that the MMSE design of time multiplexing codes is given by (9), where $\mathbf{A}$ and $\mathbf{B}$ are now of size $M \times N$ rather than $N \times N$. Following derivations similar to those in (21), (19), (23) and (24), the design of $\mathbf{A}, \mathbf{B} \in \mathbb{R}^{M \times N}$ turns out to solve the following optimization problem

$$
\begin{aligned}
& \left\{\mathbf{A}^{\star}, P^{\star}\right\} \\
& =\arg \min _{\mathbf{A} \in \mathbb{R}^{M \times N}, P \in \mathbb{R}} \operatorname{Tr}\left(-\left(\mathbf{A R} \mathbf{A}^{T}+\boldsymbol{\Sigma}_{P}\right)^{-1} \mathbf{A} \mathbf{R}^{2} \mathbf{A}^{T}+\mathbf{R}\right) \\
& \text { s.t. } 0 \preceq \operatorname{vec}(\mathbf{A}) \preceq \mathbf{1}_{M N}, \mathbf{A} \mathbf{1}_{N}=P \mathbf{1}_{M} .
\end{aligned}
$$

The optimal demultiplexing matrix is expressed as a function of $\mathbf{A}^{\star}$ and $P^{\star}$, given by

$$
\mathbf{B}^{\star}=\left(\mathbf{A}^{\star} \mathbf{R}\left(\mathbf{A}^{\star}\right)^{T}+\boldsymbol{\Sigma}_{P^{\star}}\right)^{-1} \mathbf{A}^{\star} \mathbf{R} .
$$

Likewise, applying the approximation (25) of the objective function of problem (45) one can easily come up with

$$
\begin{aligned}
& \min _{\mathbf{A} \in \mathbb{R}^{M \times N}, P \in \mathbb{R}} \operatorname{Tr}\left(\left(\mathbf{A R} \mathbf{A}^{T}+\boldsymbol{\Sigma}_{P}\right)^{-1} \boldsymbol{\Sigma}_{P}\right) \\
& \text { s.t. } \quad \mathbf{0} \preceq \operatorname{vec}(\mathbf{A}) \preceq \mathbf{1}_{M N}, \mathbf{A} \mathbf{1}_{N}=P \mathbf{1}_{M} .
\end{aligned}
$$

Since this problem has an identical structure to (27), following the formulations in Section III-A, one can easily apply Algorithm 1 to solve problem (47).

\section{B. Lagrangian Method for Problem (39)}

Problem (39) can be alternatively written in the following equivalent form:

$$
\min _{\mathbf{F} \in \mathcal{F}_{P}}\left\{\min _{\mathbf{G}^{T} \mathbf{F}=\mathbf{I}_{N}} \operatorname{Tr}\left(\mathbf{G}^{T} \boldsymbol{\Lambda}_{P} \mathbf{G}\right)\right\} .
$$

The Lagrangian associated with the inner problem of (48) is expressed as

$$
\mathcal{L}(\mathbf{G}, \mathbf{Z})=\operatorname{Tr}\left(\mathbf{G}^{T} \boldsymbol{\Lambda}_{P} \mathbf{G}\right)+\operatorname{Tr}\left(\left(\mathbf{G}^{T} \mathbf{F}-\mathbf{I}_{N}\right) \mathbf{Z}\right),
$$

where $\mathbf{Z} \in \mathbb{R}^{N \times N}$ denotes the Lagrange multiplier. Setting the partial derivatives of $\mathcal{L}(\mathbf{G}, \mathbf{Z})$ with respect to $\mathbf{G}$ and $\mathbf{Z}$ to zero, respectively, gives rise to

$$
\begin{aligned}
& \frac{\partial \mathcal{L}(\mathbf{G}, \mathbf{Z})}{\partial \mathbf{G}}=\mathbf{0} \Leftrightarrow \mathbf{G}=-\boldsymbol{\Lambda}_{P}^{-1} \mathbf{F} \mathbf{Z} / 2, \\
& \frac{\partial \mathcal{L}(\mathbf{G}, \mathbf{Z})}{\partial \mathbf{Z}}=\mathbf{0} \Leftrightarrow \mathbf{G}^{T} \mathbf{F}=\mathbf{I}_{N} .
\end{aligned}
$$

By (50) and (51), we can easily come up with

$$
\mathbf{Z}=-2\left(\mathbf{F}^{T} \boldsymbol{\Lambda}_{P}^{-1} \mathbf{F}\right)^{-1}
$$

Hence, substituting (52) into (50) yields $\mathbf{G}=$ $\boldsymbol{\Lambda}_{P}^{-1} \mathbf{F}\left(\mathbf{F}^{T} \boldsymbol{\Lambda}_{P}^{-1} \mathbf{F}\right)^{-1}$.

\section{ACKNOWLEDGMENT}

The authors wish to thank Mr. N. Ratner and Dr. Y. Y. Schechner for providing the Matlab code for the method in [10].

\section{REFERENCES}

[1] M. Harwit and N. J. A. Sloane, Hadamard Transform Optics. New York, NY, USA: Academic, 1979.

[2] Y. Y. Schechner, S. K. Nayar, and P. N. Belhumeur, "A theory of multiplexed illumination," in Proc. IEEE Int.Conf. Comput. Vision, 2003, pp. 808-815.

[3] J. Gu, T. Kobayashi, M. Gupta, and S. K. Nayar, "Multiplexed illumination for scene recovery in the presence of global illumination," in Proc. IEEE Int. Conf. Comput. Vision, 2011, pp. 691-698.

[4] Y. Y. Schechner, S. K. Nayar, and P. N. Belhumeur, "Multiplexing for optimal lighting," IEEE Trans. Pattern Anal. Mach. Intell., vol. 29, no. 8, pp. 1339-1354, 2007.

[5] A. Wenger, A. Gardner, C. Tchou, J. Unger, T. Hawkins, and P. Debevec, "Performance relighting and reflectance transformation with time-multiplexed illumination," ACM Trans. Graph. (Proc. SIGGRAPH), vol. 24, no. 3, pp. 756-764, 2005.

[6] H. Kim, B. Wilburn, and M. Ben-Ezra, "Photometric stereo for dynamic surface orientations," in Proc. Eur. Conf. Comput. Vision, 2010, pp. $59-72$.

[7] G. Fyffe, X. Yu, and P. Debevec, "Single-shot photometric stereo by spectral multiplexing," in Proc. IEEE Int. Conf. Comput. Photog., 2011, pp. 1-6.

[8] A. Wagner, J. Wright, A. Ganesh, Z. Zhou, H. Mobahi, and Y. Ma, "Towards a practical face recognition system: Robust alignment and illumination by sparse representation," IEEE Trans. Pattern Anal. Mach. Intell., vol. 34, no. 2, pp. 372-386, 2012.

[9] A. Wutting, "Optimal transformations for optical multiplex measurements in the presence of photon noise," Appl. Optics, vol. 44, no. 14, pp. 2710-2719, 2005.

[10] N. Ratner and Y. Y. Schechner, "Illumination multiplexing within fundamental limits," in Proc. IEEE Conf. Comput. Vision Pattern Recog., 2007, pp. $1-8$

[11] N. Ratner, Y. Y. Schechner, and F. Goldberg, "Optimal multiplexed sensing: Bounds, conditions and a graph theory link," Optics Express, vol. 15 , no. 25 , pp. $17072-17079,2007$.

[12] M. Alterman, Y. Y. Schechner, and A. Weiss, "Multiplexed fluorescence unmixing," in Proc. IEEE Int. Conf. Comput. Photog., 2010, pp. $1-8$. 
[13] J.-I. Park, M.-H. Lee, M. D. Grossberg, and S. K. Nayar, "Multispectral imaging using multiplexed illumination," in Proc. IEEE Int. Conf. Comput. Vision, 2007, pp. 1-8.

[14] B. D. Decker, J. Kautz, T. Mertens, and P. Bekaert, "Capturing multiple illumination conditions using time and color multiplexing," in Proc. IEEE Conf. Comput. Vision Pattern Recog., 2009, pp. 2536-2543.

[15] T.-H. Chan, K. Jia, E. Wycoff, C.-Y. Chi, and Y. Ma, "Towards optimal design of time and color multiplexing codes," in Proc. Eur. Conf. Comput. Vision, 2012, pp. 485-498.

[16] C. Liu, W. T. Freemand, R. Szeliski, and S. B. Kang, "Noise estimation from a single image," in Proc. IEEE Conf. Comput.Vision Pattern Recog., 2006, pp. 901-908.

[17] R. A. Horn and C. R. Johnson, Matrix Anal. . Cambridge, U.K.: Cambridge Univ. Press, 1985

[18] N. J. Higham, "Newton's method for the matrix square root," Math. Comput., vol. 46, no. 174, pp. 537-549, 1986.

[19] M. Grant and S. Boyd, "CVX: Matlab software for disciplined convex programming, version 1.21," Apr. 2011.

[20] B. Fares, D. Noll, and P. Apkarian, "Robust control via sequential semidefinite programming," SIAM J. Control Optimization, vol. 40, pp. 1791-1820, 2002.

[21] Q. T. Dinh and M. Diehl, "Local convergence of sequential convex programming for nonlinear programming," in Chapter of Recent Advances in Optimization and Its Application in Engineering, M. Diehl, F. Glineur, W. E. Jarlebring, and Michiels, Eds. Berlin, Germany: Springer-Verlag, 2010, pp. 93-102.

[22] R. Fletcher, Practical Methods of Optimization. New York, NY, USA: Wiley, 1987.

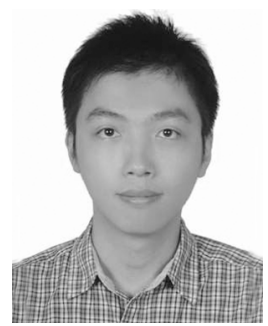

Tsung-Han Chan received the B.S. degree from the Department of Electrical Engineering, Yuan $\mathrm{Ze}$ University, Taoyuan, Taiwan, in 2004 and the Ph.D. degree from the Institute of Communications Engineering, National Tsing Hua University (NTHU), Hsinchu, Taiwan, in 2009.

$\mathrm{He}$ is currently a Senior R\&D Engineer with Sunplus Technology Company, Hsinchu, Taiwan. He was a visiting Doctoral Graduate Research Assistant with Virginia Polytechnic Institute and State University, Arlington, VA, USA, in 2008, and a Postdoctoral Research Fellow with NTHU from 2009 to 2012. He was also a Research Scientist at Advanced Digital Sciences Center, Singapore from 2012 to 2013. His research interests are in image processing and convex optimization, with a recent emphasis on computer vision, hyperspectral remote sensing and dynamic medical imaging applications.

He was a co-recipient of a WHISPERS 2011 Best Paper Award.

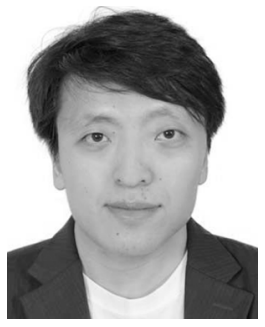

Kui Jia received the B.Eng. degree in marine engineering from Northwestern Polytechnical University, China, in 2001, the M.Eng. degree in electrical and computer engineering from National University of Singapore in 2003, and the Ph.D. degree in computer science from Queen Mary, University of London, London, U.K., in 2007.

$\mathrm{He}$ is currently a Research Scientist at Advanced Digital Sciences Center, Singapore. His research interests are in computer vision, machine learning, and image processing.

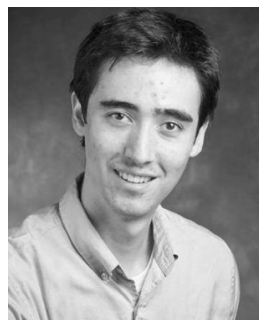

Eliot Wycoff received the B.S. degree in applied mathematics from Columbia University, New York, NY, USA, in 2011. He is currently working toward a Master's degree in aerospace engineering at the University of Illinois at Urbana-Champaign, Urbana, IL, USA, where his research focus is on global navigation satellite systems (GNSS) and cooperative positioning.

From 2011 to 2013, he was a Software Engineer at Singapore's Advanced Digital Sciences Center where he gained exposure to topics in computer vision.

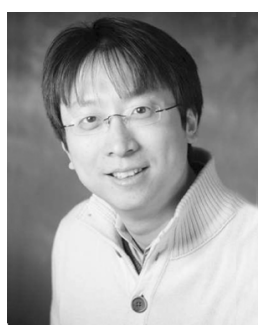

Yi Ma received the bachelor's degree in automation and applied mathematics from Tsinghua University, Beijing, in 1995. He received the M.S. degree in electrical engineering and computer science (EECS) in 1997, the M.A. degree in mathematics in 2000, and the Ph.D. degree in EECS in 2000, all from the University of California, Berkeley, CA, USA.

From 2000 to 2011, he was with the faculty of the Electrical and Computer Engineering Department, University of Illinois at Urbana-Champaign (UIUC), Urbana, IL, USA. Currently, he is a Principal Researcher at Microsoft Research, and an Adjunct Professor at UIUC. His main research interests include computer vision and high-dimensional data analysis.

$\mathrm{He}$ is a Fellow of IEEE and a member of ACM. He was the recipient of the David Marr Best Paper Prize at the International Conference on Computer Vision in 1999 and Honorable Mention for the Longuet-Higgins Best Paper Award at the European Conference on Computer Vision in 2004. He received the CAREER Award from the USA National Science Foundation in 2004 and the Young Investigator Program Award from the USA Office of Naval Research in 2005. He was an associate editor for IEEE TRANSACTIONS ON PATTERN ANALYSIS AND MACHINE INTELLIGENCE from 2007 to 2011, and is currently an associate editor for the International Journal of Computer Vision, IEEE TRANSACTIONS ON INFORMATION THEORY, SIAM Journal on Imaging Sciences, and the IMA Journal on Information and Inference. 